\title{
Alleviating the non-ultralocality of coset $\sigma$-models through a generalized Faddeev-Reshetikhin procedure
}

\author{
F. Delduc, ${ }^{a}$ M. Magro $^{a}$ and B. Vicedo ${ }^{b}$ \\ ${ }^{a}$ Laboratoire de Physique, ENS Lyon et CNRS UMR 5672, Université de Lyon, \\ 46, allée d'Italie, 69364 LYON Cedex 0\%, France \\ ${ }^{b}$ Department of Mathematics, University of York, \\ Heslington, York, YO10 5DD, United Kingdom \\ E-mail: Francois.Delduc@ens-lyon.fr, Marc.Magro@ens-lyon.fr, \\ Benoit.Vicedo@gmail.com
}

AbstRAct: The Faddeev-Reshetikhin procedure corresponds to a removal of the nonultralocality of the classical SU(2) principal chiral model. It is realized by defining another field theory, which has the same Lax pair and equations of motion but a different Poisson structure and Hamiltonian. Following earlier work of M. Semenov-Tian-Shansky and A. Sevostyanov, we show how it is possible to alleviate in a similar way the non-ultralocality of symmetric space $\sigma$-models. The equivalence of the equations of motion holds only at the level of the Pohlmeyer reduction of these models, which corresponds to symmetric space sine-Gordon models. This work therefore shows indirectly that symmetric space sine-Gordon models, defined by a gauged Wess-Zumino-Witten action with an integrable potential, have a mild non-ultralocality. The first step needed to construct an integrable discretization of these models is performed by determining the discrete analogue of the Poisson algebra of their Lax matrices.

KeYwords: Lattice Integrable Models, Integrable Field Theories, Sigma Models

ARXiv EPRINT: 1204.0766 


\section{Contents}

1 Introduction $\quad 2$

2 Generalizing the Faddeev-Reshetikhin bracket 4

2.1 Setup of the procedure 4

2.1.1 Problem with non-ultralocality 4

2.1.2 Algebraic formulation 5

2.2 Principal chiral model 6

2.2.1 Original bracket 6

$\begin{array}{lll}2.2 .2 & \text { Ultralocal bracket } & 7\end{array}$

2.3 Symmetric space $\sigma$-model $\quad 8$

2.3.1 Original bracket 8

2.3.2 Mildly non-ultralocal bracket 9

2.4 Compatibility of the Poisson brackets 11

3 Dynamics and Hamiltonian 11

$\begin{array}{ll}3.1 & \text { Original dynamics and gauge invariance } \\ \end{array}$

3.2 Casimirs of the new Poisson bracket and Pohlmeyer reduction 12

$\begin{array}{lll}3.3 & \text { Lifting to } G & 14\end{array}$

$\begin{array}{lll}3.3 .1 & \text { Poisson brackets } & 14\end{array}$

$\begin{array}{lll}\text { 3.3.2 } & \text { Reduced original dynamics } & 15\end{array}$

$\begin{array}{ll}\text { 3.3.3 Gauge invariances and anomalies } & 16\end{array}$

$\begin{array}{ll}3.4 & \text { New Hamiltonian } \\ & 16\end{array}$

$\begin{array}{lll}\text { 3.4.1 Partial gauge fixing on equations of motion } & 16\end{array}$

$\begin{array}{lll}3.4 .2 & \text { Hamiltonian } & 18\end{array}$

$\begin{array}{lll}3.4 .3 & \text { Lax pair } & 18\end{array}$

4 Towards a lattice discretization $\quad 19$

$\begin{array}{lll}4.1 & \text { Generalized Gauss decomposition } & 19\end{array}$

$\begin{array}{lll}4.2 & \text { Lattice algebra } & 20\end{array}$

5 Comments and conclusion $\quad 23$

$\begin{array}{ll}\text { A Notations } & 25\end{array}$ 


\section{Introduction}

The Quantum Inverse Scattering Method (QISM) [1-3] provides a very general and successful framework for establishing and studying the quantum integrability of a broad class of $(1+1)$-dimensional quantum field theories. Yet despite its formidable success, a significant number of important quantum field theories which are believed to be quantum integrable have so far resisted its application. Some well known examples are the principal chiral field model and the symmetric space $\sigma$-model. What distinguishes these theories is that they violate one of the key assumptions behind the QISM, known as ultralocality. In fact, the difficulty in dealing with these so called non-ultralocal theories is already apparent classically. Indeed, the requirement of ultralocality classically means that the Poisson bracket of the Lax matrix with itself does not depend on derivatives of the Dirac $\delta$-function. When this assumption fails, the computation of the Poisson bracket of the monodromy matrix becomes plagued with ambiguities. Attempting to fix these ambiguities leads to a bracket that doesn't satisfy the Jacobi identity $[4,5]$. And although a proof of classical integrability is still possible in this case, the lack of a well defined Poisson bracket of monodromies severely hinders the introduction of an integrable lattice discretization for these models.

For the SU(2) principal chiral model, however, the situation is slightly better. Indeed, in 1986, L. Faddeev and N. Reshetikhin put forward an interesting proposal in [6] for circumventing the problem of non-ultralocality in this model. The first step taken in [6] was to replace the problematic non-ultralocal Poisson brackets by ultralocal ones. In doing so, the Hamiltonian also needs to be modified in order to reproduce the classical dynamics of the SU(2) principal chiral model. As a matter of fact, the new ultralocal Poisson brackets turn out to be degenerate, which means that they can only be used to reproduce a reduction of the original dynamics where the Casimirs have been set to constants. Nevertheless, given this classically equivalent description of the model in terms of an ultralocal Poisson structure, it could be quantized within the QISM.

It is natural to ask whether such a method can be generalized to other non-ultralocal models as well. Indeed, if this were possible, the Faddeev-Reshetikhin procedure may provide a consistent way of treating more general non-ultralocal theories and fitting them into the general scheme of the QISM. The purpose of this article is to initiate such a program by generalizing the first steps of the Faddeev-Reshetikhin procedure to the case of symmetric space $\sigma$-models. Specifically, we shall propose a modification of the Poisson brackets and Hamiltonian of these models in the spirit of [6] which will lead to a well defined lattice Poisson algebra.

The first task of determining the modified Poisson brackets is a kinematical one. As we shall see, unlike the case of the principal chiral model, it won't be possible to completely do away with the non-ultralocality in the Poisson brackets of coset $\sigma$-models. It will nevertheless be possible to alleviate their non-ultralocality in the following sense. It was shown by M. Semenov-Tian-Shansky and A. Sevostyanov in [7] that there exists a natural non-ultralocal Poisson structure on these models which, after regularization, does admit an integrable lattice discretization of the general form identified in [36, 37]. We shall refer to this special form of non-ultralocality as being mild. Our generalization of the Faddeev- 
Reshetikhin modification of the Poisson bracket will therefore be to replace the original non-ultralocal Poisson structure by this milder non-ultralocal one.

The second task of determining the corresponding Hamiltonian is a dynamical one. Just as for the $\mathrm{SU}(2)$ principal chiral model [6], it turns out that the modified Poisson brackets are degenerate. Since the corresponding set of Casimirs will necessarily remain constant in time, this means that we can only reproduce a reduction of the original dynamics. Quite remarkably, the specific form of the Casimirs of the modified Poisson brackets leads naturally to performing a Pohlmeyer reduction [8] of the symmetric space $\sigma$-model. In other words, the equations of motion that we shall be able to reproduce using the modified Poisson brackets are precisely those of the Pohlmeyer reduction of the original $\sigma$-model. In fact more is true. Recall that the equations of motion obtained by this reduction identify with those of a symmetric space sine-Gordon theory [9-16], the Lagrangian formulation of which corresponds to a gauged Wess-Zumino-Witten model with an integrable potential [17] (see also $[18,19]$ ). An important result of the present work is that the canonical Poisson brackets associated with this latter model [20] precisely coincide with the alleviated non-ultralocal Poisson brackets of the coset $\sigma$-model. In particular, this shows indirectly that the non-ultralocality of gauged WZW models with an integrable potential is mild.

The plan of this article is as follows. After a short reminder of the problem of nonultralocality and its formulation within the $R$-matrix approach [21-24], in section 2 we recast the initial step of the Faddeev-Reshetikhin procedure in a general algebraic framework that enables a systematic and practical determination of the new Poisson brackets. This is achieved by first generalizing the Faddeev-Reshetikhin Poisson brackets to the principal chiral model on a generic Lie group before extending these ideas to symmetric space $\sigma$-models. In particular, we show in both cases that these modified Poisson brackets are compatible with the original ones.

In section 3, we discuss the dynamics of the coset $\sigma$-model with respect to the new Poisson brackets. We show that fixing the values of all the Casimirs of these degenerate brackets amounts to doing a Pohlmeyer reduction of the $\sigma$-model. As usual, the reduced field equations have separate left and right gauge invariances which have to be partially fixed to the diagonal gauge invariance before the model can be described in the Hamiltonian framework. The resulting dynamics and Poisson brackets of the reduced fields are those of a gauged WZW model with a potential term. Finally, we also write down the Lax matrix of the reduced model.

Section 4 is devoted to the first step towards discretization. Following [7], we give an integrable lattice discretization of the Poisson brackets as in [36, 37]. The construction uses an arbitrary solution of the modified Yang Baxter equation on a finite dimensional Lie algebra. The Poisson bracket of the Lax matrix on the lattice are given, from which we deduce the regularized Poisson bracket of the monodromy matrix. Taking the continuum limit we then find that the former bracket correctly reproduces the Poisson bracket of the Lax matrix of the continuum theory.

Finally, some comments, a conclusion and some outlooks are gathered in section 5 . For general notations we refer the reader to the appendix. 


\section{Generalizing the Faddeev-Reshetikhin bracket}

\subsection{Setup of the procedure}

\subsubsection{Problem with non-ultralocality}

To begin with let us briefly recall what is meant by non-ultralocality in classical integrable field theory and why this property leads to a severe obstacle for quantization. Consider a classical integrable field theory on the circle whose field content is specified by a Lax matrix $\mathcal{L}(\sigma, \lambda)$. We let $\sigma \in S^{1}$ be a parameter on the circle and $\lambda \in \mathbb{C}$ be the so called spectral parameter. It is well known [5] that a fairly general form of the Poisson bracket of $\mathcal{L}(\sigma, \lambda)$ with itself which will guarantee complete integrability of the theory is ${ }^{1}$

$$
\begin{aligned}
\left\{\mathcal{L}_{\underline{\mathbf{1}}}(\sigma, \lambda), \mathcal{L}_{\underline{\mathbf{2}}}\left(\sigma^{\prime}, \mu\right)\right\}=\left[r_{\underline{\mathbf{1 2}}}(\lambda, \mu), \mathcal{L}_{\underline{\mathbf{1}}}(\sigma, \lambda)+\mathcal{L}_{\underline{\mathbf{2}}}(\sigma, \mu)\right] \delta_{\sigma \sigma^{\prime}} \\
+\left[\underline{\underline{\mathbf{1 2}}}(\lambda, \mu), \mathcal{L}_{\underline{\mathbf{1}}}(\sigma, \lambda)-\mathcal{L}_{\underline{\mathbf{2}}}(\sigma, \mu)\right] \delta_{\sigma \sigma^{\prime}}+2 s_{\underline{\mathbf{1 2}}}(\lambda, \mu) \delta_{\sigma \sigma^{\prime}}^{\prime}
\end{aligned}
$$

Throughout we let $\delta_{\sigma \sigma^{\prime}}$ denote the Dirac $\delta$-function and set $\delta_{\sigma \sigma^{\prime}}^{\prime}=\partial_{\sigma} \delta_{\sigma \sigma^{\prime}}$. The theory is then said to be ultralocal if $s_{\mathbf{1 2}}=0$ and non-ultralocal otherwise. In particular, nonultralocal theories are characterized by the presence of the $\delta_{\sigma \sigma^{\prime}}^{\prime}$ term in (2.1).

A standard approach to quantizing an integrable field theory [1-3] begins by introducing a lattice regularization to handle the UV divergences of the quantum theory. To discretize the classical integrable field theory, we start by breaking up the circle at a finite set of points $\sigma_{n} \in S^{1}, n=1, \ldots, N$. The lattice Lax matrix $\mathcal{L}^{n}(\lambda)$ is then defined to be the parallel transporter from the site $\sigma_{n}$ to the next site $\sigma_{n+1}$, namely

$$
\mathcal{L}^{n}(\lambda)=P \overleftarrow{\exp } \int_{\sigma_{n}}^{\sigma_{n+1}} \mathcal{L}(\sigma, \lambda) d \sigma
$$

By using the Leibniz rule one can reduce the computation of the Poisson bracket between $\mathcal{L}^{n}(\lambda)$ and $\mathcal{L}^{m}(\mu)$ to a double integral involving the Poisson bracket (2.1). When dealing with ultralocal theories for which $s_{\underline{12}}=0$, the substitution of (2.1) into this double integral is unambiguous and leads to the following ultralocal lattice algebra

$$
\left\{\mathcal{L}_{\underline{\mathbf{1}}}^{n}(\lambda), \mathcal{L}_{\underline{\mathbf{2}}}^{m}(\mu)\right\}=\left[r_{\underline{\mathbf{1 2}}}(\lambda, \mu), \mathcal{L}_{\underline{\mathbf{1}}}^{n}(\lambda) \mathcal{L}_{\underline{\mathbf{2}}}^{m}(\mu)\right] \delta_{m n},
$$

where $\delta_{m n}$ is the Kronecker symbol. The quantization of the lattice algebra (2.2) constitutes the starting point for the QISM.

However, when $s_{\underline{12}} \neq 0$, the presence of the $\delta_{\sigma \sigma^{\prime}}^{\prime}$ term in (2.1) leads to ambiguities when evaluating the double integral, the reason being that the Poisson bracket of parallel transporters is not well defined whenever any two of their end-points coincide [5]. As a result, in the case of a generic non-ultralocal theory, the $\mathcal{L}^{n}(\lambda)$ do not have well defined Poisson brackets.

\footnotetext{
${ }^{1}$ For later convenience we have departed from the usual convention by changing the overall sign of $s_{\mathbf{1 2}}$.
} 


\subsubsection{Algebraic formulation}

In order to generalize the Faddeev-Reshetikhin procedure it will be essential to isolate the root of non-ultralocality. In view of this it is extremely useful to phrase the latter in a somewhat abstract setting [21]. In this setting, the integrable field theories we shall be considering are associated with a set

$$
\widehat{(\mathfrak{f}}, \mathcal{L}, R, \varphi),
$$

where $\widehat{\mathfrak{f}}$ is a loop algebra, the Lax matrix $\mathcal{L}$ is a map from $S^{1}$ to $\widehat{\mathfrak{f}}$ and $R$ is an $R$-matrix, i.e. an element of End $\widehat{\mathfrak{f}}$ satisfying the modified classical Yang-Baxter equation (mCYBE) on $\widehat{\mathfrak{f}}$

$$
\forall X, Y \in \widehat{\mathfrak{f}}, \quad[R X, R Y]-R([R X, Y]+[X, R Y])+\omega[X, Y]=0
$$

with $\omega=1$. Equation (2.4) ensures (for any value of $\omega$ ) that the so called $R$-bracket, defined by

$$
[X, Y]_{R}=[R X, Y]+[X, R Y]
$$

is a Lie bracket on $\widehat{\mathfrak{f}}$. The last input in $(2.3)$ is a formal Laurent series $\varphi(\lambda) \in \mathbb{C}((\lambda))$, called the twist, specifying an inner product on $\widehat{\mathfrak{f}}$. Fixing a non singular invariant inner product $\langle\cdot, \cdot\rangle$ on $\mathfrak{f}$, the latter is defined in terms of $\varphi$ by taking the following residue

$$
(X, Y)_{\varphi}=\operatorname{res}_{\lambda=0} d \lambda \varphi(\lambda)\langle X(\lambda), Y(\lambda)\rangle
$$

for any $X, Y \in \widehat{\mathfrak{f}}$. This is sometimes referred to as the twisted inner product on $\widehat{\mathfrak{f}}$.

The Poisson brackets (2.1) of the corresponding integrable field theory can now be expressed in terms of the data (2.3) as follows. We equip the space $C^{\infty}\left(S^{1}, \widehat{\mathfrak{f}}\right)$, to which $\mathcal{L}$ belongs, with the following inner product and cocycle,

$$
((\mathcal{X}, \mathcal{Y}))_{\varphi}=\int_{S^{1}} d \sigma(\mathcal{X}(\sigma), \mathcal{Y}(\sigma))_{\varphi}, \quad \omega_{\varphi}(\mathcal{X}, \mathcal{Y})=\int_{S^{1}} d \sigma\left(\mathcal{X}(\sigma), \partial_{\sigma} \mathcal{Y}(\sigma)\right)_{\varphi}
$$

The Poisson bracket between any two functions $f, g$ of the Lax matrix can then be written as

$$
\{f, g\}(\mathcal{L})=\left(\left(\mathcal{L},\left[R d_{\varphi} f, d_{\varphi} g\right]+\left[d_{\varphi} f, R d_{\varphi} g\right]\right)\right)_{\varphi}+\left(\omega_{\varphi}\left(R d_{\varphi} f, d_{\varphi} g\right)+\omega_{\varphi}\left(d_{\varphi} f, R d_{\varphi} g\right)\right)
$$

The subscript $\varphi$ on the differential is used to indicate that $d_{\varphi} f(\mathcal{L})$ is defined relative to the inner product $(2.7)$, in other words

$$
\left(\left(\mathcal{X}, d_{\varphi} f(\mathcal{L})\right)\right)_{\varphi}=\left.\frac{d}{d t}\right|_{t=0} f(\mathcal{L}+t \mathcal{X}) .
$$

This Poisson bracket is merely the Kostant-Kirillov bracket on the central extension of $C^{\infty}\left(S^{1}, \widehat{\mathfrak{f}}\right)$, defined by the cocycle $\omega_{\varphi}$, associated with the $R$-bracket $(2.5)$. To bring it to a more recognizable form comparable with (2.1) we restrict attention to linear functions $f, g$ of $\mathcal{L} \in C^{\infty}\left(S^{1}, \widehat{\mathfrak{f}}\right)$ and use tensor notation. Letting $R^{*}$ denote the adjoint of $R$ with respect to $(2.6)$ one finds

$$
\left\{\mathcal{L}_{\underline{1}}(\sigma), \mathcal{L}_{\underline{\mathbf{2}}}\left(\sigma^{\prime}\right)\right\}=\left[R_{\underline{\mathbf{1 2}}}, \mathcal{L}_{\underline{\mathbf{1}}}(\sigma)\right] \delta_{\sigma \sigma^{\prime}}-\left[R_{\underline{\mathbf{1 2}}}^{*}, \mathcal{L}_{\underline{\mathbf{2}}}(\sigma)\right] \delta_{\sigma \sigma^{\prime}}+\left(R_{\underline{\mathbf{1 2}}}+R_{\underline{\mathbf{1 2}}}^{*}\right) \delta_{\sigma \sigma^{\prime}}^{\prime} .
$$


We refer the reader for instance to [24] for details but simply note here that the term in $\delta_{\sigma \sigma^{\prime}}^{\prime}$ comes precisely from the cocycle $\omega_{\varphi}$. The Poisson bracket (2.9) is then identified with (2.1) if we define the matrices $r_{\underline{12}}$ and $s_{\underline{12}}$ to be the kernels of the skew-symmetric and symmetric parts of $R$ respectively,

$$
r=\frac{1}{2}\left(R-R^{*}\right) \quad \text { and } \quad s=\frac{1}{2}\left(R+R^{*}\right) .
$$

It is apparent from (2.10) that ultralocal theories correspond to the situation where the $R$-matrix is skew-symmetric with respect to the inner product, i.e. $R^{*}=-R$.

As we will see below, on an abstract level the procedure of [6] consists in keeping the same loop algebra, Lax matrix and $R$-matrix but changing the inner product in such a way that $R^{*}=-R$ with respect to the new inner product. This has the desired effect of replacing the problematic non-ultralocal Poisson bracket of the Lax matrix of the SU(2) principal chiral model by an ultralocal one. When expressed in terms of the dynamical fields, this latter Poisson bracket corresponds precisely to the modified bracket of [6]. In fact, we will show this more generally by working with the principal chiral model on a generic Lie group. We then generalize these ideas to symmetric space $\sigma$-models.

\subsection{Principal chiral model}

\subsubsection{Original bracket}

We start by identifying the set $(\widehat{\mathfrak{f}}, \mathcal{L}, R, \varphi)$ in the case of the principal chiral model on a Lie group $F$. The first element is simply the loop algebra $\widehat{\mathfrak{f}}=\mathfrak{f} \otimes \mathbb{C}((\lambda))$ of formal Laurent series with coefficients in $\mathfrak{f}=\operatorname{Lie}(F)$. In terms of the usual components $\left(j_{0}, j_{1}\right)$ of the current $j$ taking values in $\mathfrak{f}$, the Lax matrix of the principal chiral model is given by

$$
\mathcal{L}=\frac{1}{1-\lambda^{2}}\left(j_{1}+\lambda j_{0}\right) .
$$

Next, the $R$-matrix is defined by choosing a pair of complementary subalgebras of $\widehat{\mathfrak{f}}$. In the obvious notation we let

$$
\widehat{\mathfrak{f}}_{\geq 0}=\mathfrak{f} \otimes \mathbb{C} \llbracket \lambda \rrbracket, \quad \widehat{\mathfrak{f}}_{<0}=\mathfrak{f} \otimes \lambda^{-1} \mathbb{C}\left[\lambda^{-1}\right],
$$

and similarly for $\widehat{\mathfrak{f}}_{>0}$ and $\widehat{\mathfrak{f}} \leq 0$. Let $\pi_{\geq 0}, \pi_{<0}, \pi_{>0}$ and $\pi_{\leq 0}$ be the projections of $\widehat{\mathfrak{f}}$ onto these respective subalgebras. For later purposes let us also introduce the projection $\pi_{0}$ onto the constant subalgebra $\mathfrak{f} \subset \widehat{\mathfrak{f}}$. The standard $R$-matrix can now be defined as

$$
R=\pi_{\geq 0}-\pi_{<0} .
$$

Finally, the inner product (2.6) on $\widehat{\mathfrak{f}}$ is given by the following choice of twist

$$
\varphi(\lambda)=2\left(1-\frac{1}{\lambda^{2}}\right) .
$$

As a result of this twist, the $R$-matrix (2.12) is not skew-symmetric. Indeed, the adjoint $R^{*}$ of $R$ can be computed explicitly as

$$
\begin{aligned}
\operatorname{res}_{\lambda=0} d \lambda \varphi(\lambda)\langle R(X(\lambda)), Y(\lambda)\rangle & =\operatorname{res}_{\lambda=0} d \lambda\left\langle\pi_{\geq 0}(X(\lambda))-\pi_{<0}(X(\lambda)), \varphi(\lambda) Y(\lambda)\right\rangle, \\
& =\operatorname{res}_{\lambda=0} d \lambda\left\langle X(\lambda), \pi_{<0}(\varphi(\lambda) Y(\lambda))-\pi_{\geq 0}(\varphi(\lambda) Y(\lambda))\right\rangle, \\
& =-\operatorname{res}_{\lambda=0} d \lambda \varphi(\lambda)\left\langle X(\lambda), \varphi(\lambda)^{-1} R(\varphi(\lambda) Y(\lambda))\right\rangle,
\end{aligned}
$$


from which we deduce that

$$
R^{*}=-\tilde{\varphi}^{-1} \circ R \circ \tilde{\varphi}
$$

where $\tilde{\varphi}$ denotes the multiplication by $\varphi(\lambda)$.

The expression (2.8) is very useful if one wants to derive the Poisson brackets of the currents from those of the Lax matrix. Indeed, consider $x \in \mathfrak{f}$. Then $\lambda x$ is in $\widehat{\mathfrak{f}}$ and we have

$$
\left(\left(\mathcal{L},-\frac{1}{2} \lambda x \cdot \delta_{\sigma}\right)\right)_{\varphi}=-\int_{S^{1}} d \sigma^{\prime} \delta_{\sigma \sigma^{\prime}} \operatorname{res}_{\lambda=0} d \lambda \frac{\varphi(\lambda)}{1-\lambda^{2}}\left\langle j_{1}\left(\sigma^{\prime}\right)+\lambda j_{0}\left(\sigma^{\prime}\right), \frac{1}{2} \lambda x\right\rangle=\left\langle j_{1}(\sigma), x\right\rangle .
$$

We define then two linear functions for any $x \in \mathfrak{f}$ as

$$
j_{\sigma, x}^{0}: \mathcal{L} \mapsto\left(\left(\mathcal{L},-\frac{1}{2} x \cdot \delta_{\sigma}\right)\right)_{\varphi}=\left\langle j_{0}(\sigma), x\right\rangle, \quad j_{\sigma, x}^{1}: \mathcal{L} \mapsto\left(\left(\mathcal{L},-\frac{1}{2} \lambda x \cdot \delta_{\sigma}\right)\right)_{\varphi}=\left\langle j_{1}(\sigma), x\right\rangle .
$$

In particular, since these functions are linear we have

$$
d_{\varphi} j_{\sigma, x}^{0}=-\frac{1}{2} x \cdot \delta_{\sigma} \quad \text { and } \quad d_{\varphi} j_{\sigma, x}^{1}=-\frac{1}{2} \lambda x \cdot \delta_{\sigma} .
$$

We can now extract the Poisson brackets of the fields $j_{0}, j_{1}$ from (2.8). For instance,

$$
\begin{aligned}
\left\langle\left\{j_{0 \underline{1}}(\sigma), j_{1 \underline{\mathbf{1}}}\left(\sigma^{\prime}\right)\right\}, x_{\underline{\mathbf{1}}} y_{\underline{\mathbf{2}}}\right\rangle_{\underline{\mathbf{1 2}}=} & \left\{j_{\sigma, x}^{0}, j_{\sigma^{\prime}, y}^{1}\right\}(\mathcal{L}), \\
= & \frac{1}{4}(\mathcal{L},[R(x), \lambda y]+[x, R(\lambda y)])_{\varphi} \delta_{\sigma \sigma^{\prime}} \\
& +\frac{1}{4}\left((R(x), \lambda y)_{\varphi}+(x, R(\lambda y))_{\varphi}\right) \delta_{\sigma \sigma^{\prime}}^{\prime} \\
= & \frac{1}{2}(\mathcal{L}, \lambda[x, y])_{\varphi} \delta_{\sigma \sigma^{\prime}}+\frac{1}{2}(x, \lambda y)_{\varphi} \delta_{\sigma \sigma^{\prime}}^{\prime} \\
= & -\left\langle j_{1}(\sigma),[x, y]\right\rangle \delta_{\sigma \sigma^{\prime}}-\langle x, y\rangle \delta_{\sigma \sigma^{\prime}}^{\prime}
\end{aligned}
$$

The bracket $\left\{j_{0}, j_{1}\right\}$ follows from this computation since $x, y \in \mathfrak{f}$ are arbitrary. The remaining brackets $\left\{j_{0}, j_{0}\right\}$ and $\left\{j_{1}, j_{1}\right\}$ are obtained in a similar way and altogether we recover the Poisson brackets of the principal chiral model

$$
\begin{aligned}
& \left\{j_{0 \underline{\underline{1}}}(\sigma), j_{0 \underline{\mathbf{2}}}\left(\sigma^{\prime}\right)\right\}=\left[C_{\underline{\mathbf{1 2}}}, j_{0 \underline{\mathbf{2}}}(\sigma)\right] \delta_{\sigma \sigma^{\prime}}, \\
& \left\{j_{0 \underline{\mathbf{1}}}(\sigma), j_{1 \underline{\mathbf{2}}}\left(\sigma^{\prime}\right)\right\}=\left[C_{\underline{\mathbf{1 2}}}, j_{1 \underline{\mathbf{2}}}(\sigma)\right] \delta_{\sigma \sigma^{\prime}}-C_{\underline{\mathbf{1 2}}} \delta_{\sigma \sigma^{\prime}}^{\prime}, \\
& \left\{j_{1 \underline{\mathbf{1}}}(\sigma), j_{1 \underline{\mathbf{1}}}\left(\sigma^{\prime}\right)\right\}=0 .
\end{aligned}
$$

\subsubsection{Ultralocal bracket}

The non-ultralocality of the model is a consequence of the fact that its $R$-matrix $(2.12)$ is not skew with respect to the inner product (2.6) with the twist (2.13). Indeed, $R$ does not commute with $\tilde{\varphi}$ and therefore

$$
R^{*}=-\tilde{\varphi}^{-1} \circ R \circ \tilde{\varphi} \neq-R .
$$

However, it is also clear that $R$ would be skew if we had used the twist function $\varphi^{\prime}=1$ instead of (2.13). This corresponds to choosing the rational inner product on $\widehat{\mathfrak{f}}$

$$
(X, Y)_{\text {rat }}=\operatorname{res}_{\lambda=0} d \lambda\langle X(\lambda), Y(\lambda)\rangle
$$


Therefore, a natural prescription for obtaining an ultralocal model is simply to replace the twisted inner product (2.6) by the rational inner product (2.17) while keeping everything else identical. In particular we don't modify the underlying loop algebra $\widehat{\mathfrak{f}}$, we keep the same Lax matrix $\mathcal{L}$ and we don't even change the $R$-matrix!

Since the $R$-matrix (2.12) is skew-symmetric with respect to the inner product (2.17), the last term in (2.8) vanishes, leaving

$$
\{f, g\}^{\prime}(\mathcal{L})=\left(\left(\mathcal{L},\left[R d_{1} f, d_{1} g\right]+\left[d_{1} f, R d_{1} g\right]\right)\right)_{\text {rat }} .
$$

To find the resulting Poisson bracket expressed in terms of the fields $j_{0}$ and $j_{1}$ themselves we must first find how to extract these from the Lax connection. The analogues of the linear functions (2.15) in the present case read

$$
j_{\sigma, x}^{\prime 0}: \mathcal{L} \mapsto\left(\left(\mathcal{L}, \lambda^{-2} x \cdot \delta_{\sigma}\right)\right)_{\mathrm{rat}}=\left\langle j_{0}, x\right\rangle, \quad j_{\sigma, x}^{\prime 1}: \mathcal{L} \mapsto\left(\left(\mathcal{L}, \lambda^{-1} x \cdot \delta_{\sigma}\right)\right)_{\mathrm{rat}}=\left\langle j_{1}, x\right\rangle .
$$

It is now straightforward to compute for example

$$
\left\langle\left\{j_{0 \underline{1}}(\sigma), j_{1 \underline{\mathbf{2}}}\left(\sigma^{\prime}\right)\right\}^{\prime}, x_{\underline{1}} y_{\underline{\mathbf{2}}}\right\rangle_{\underline{\mathbf{1 2}}}=\left\{j_{\sigma, x}^{\prime 0}, j_{\sigma^{\prime}, y}^{\prime 1}\right\}^{\prime}(\mathcal{L})=-2\left\langle j_{1}(\sigma),[x, y]\right\rangle \delta_{\sigma \sigma^{\prime}} .
$$

The other brackets between the fields can be computed similarly and the result reads

$$
\begin{aligned}
& \left\{j_{0 \underline{\mathbf{1}}}(\sigma), j_{0 \underline{\mathbf{2}}}\left(\sigma^{\prime}\right)\right\}^{\prime}=2\left[C_{\underline{\mathbf{1 2}}}, j_{0 \underline{\mathbf{2}}}(\sigma)\right] \delta_{\sigma \sigma^{\prime}}, \\
& \left\{j_{0 \underline{\mathbf{1}}}(\sigma), j_{1 \underline{\mathbf{2}}}\left(\sigma^{\prime}\right)\right\}^{\prime}=2\left[C_{\underline{\mathbf{1 2}}}, j_{1 \underline{\mathbf{2}}}(\sigma)\right] \delta_{\sigma \sigma^{\prime}}, \\
& \left\{j_{1 \underline{\mathbf{1}}}(\sigma), j_{1 \underline{\mathbf{2}}}\left(\sigma^{\prime}\right)\right\}^{\prime}=2\left[C_{\underline{\mathbf{1 2}}}, j_{0 \underline{\mathbf{2}}}(\sigma)\right] \delta_{\sigma \sigma^{\prime}} .
\end{aligned}
$$

Up to an irrelevant overall factor of 2 , this is exactly the modified Poisson structure of Faddeev-Reshetikhin introduced in [6] in the context of the SU(2) principal chiral model. Here we have rederived the same brackets for an arbitrary Lie group $F$ by following the simple prescription

$$
\widehat{(\mathfrak{f}}, \mathcal{L}, R, \varphi) \quad \longrightarrow \quad \widehat{(\mathfrak{f}}, \mathcal{L}, R, 1) \text {. }
$$

\subsection{Symmetric space $\sigma$-model}

\subsubsection{Original bracket}

The phase-space of a $\mathbb{Z}_{2}$-graded coset $\sigma$-model is parametrized by the two gradings of the field $A=A^{(0)}+A^{(1)}$ and its canonically conjugate momentum $P=P^{(0)}+P^{(1)}$, where the gradings of the Lie algebra $\operatorname{Lie}(F)=\mathfrak{f}=\mathfrak{f}^{(0)} \oplus \mathfrak{f}^{(1)}$ are defined as the eigenspaces of an involution $\sigma: \mathfrak{f} \rightarrow \mathfrak{f}$ with $\sigma^{2}=$ id. The Lax matrix of the model reads [25]

$$
\mathcal{L}=A^{(0)}+\frac{1}{2}\left(\lambda^{-1}+\lambda\right) A^{(1)}+\frac{1}{2}\left(1-\lambda^{2}\right) \Pi^{(0)}+\frac{1}{2}\left(\lambda^{-1}-\lambda\right) \Pi^{(1)},
$$

where $\Pi=\partial_{\sigma} P-[A, P]$. To describe the algebraic structure of the model we introduce the twisted loop algebra $\widehat{\mathfrak{f}}^{\sigma}$. This is the subalgebra of the loop algebra $\widehat{\mathfrak{f}}$ consisting of elements $X(\lambda)$ which are invariant under the automorphism $\hat{\sigma}(X)(\lambda)=\sigma[X(-\lambda)]$. Concretely, we have ${ }^{2}$

$$
\widehat{\mathfrak{f}}^{\sigma}=\bigoplus_{n} \mathfrak{f}^{(n)} \cdot \lambda^{n}
$$

\footnotetext{
${ }^{2}$ The number of terms with $n$ negative is arbitrary but finite. Also, in this formula and in the rest of this article, an integer between parenthesis is only considered modulo 2. Thus, $(n)$ is (0) or (1), depending on the parity of $n$.
} 
We denote by $\widehat{\mathfrak{f}}_{<0}^{\sigma}=\widehat{\mathfrak{f}}_{<0} \cap \widehat{\mathfrak{f}}^{\sigma}$ the subalgebra of $\widehat{\mathfrak{f}}^{\sigma}$ for which the direct sum is restricted to $n<0$, and similarly for $\widehat{\mathfrak{f}}_{\leq 0}^{\sigma}, \widehat{\mathfrak{f}}_{>0}^{\sigma}, \widehat{\mathfrak{f}}_{\geq 0}^{\sigma}$. By abuse of notation we shall denote the restriction of the respective projections $\pi_{<0}, \pi_{\leq 0}, \pi_{>0}$ and $\pi_{\geq 0}$ to $\widehat{f}^{\sigma}$ by the same symbol. The twisted loop algebra inherits the decomposition $\widehat{\mathfrak{f}}^{\sigma}=\widehat{\mathfrak{f}}_{<0}^{\sigma} \dot{+} \widehat{\mathfrak{f}}_{\geq 0}^{\sigma}$ from $\widehat{\mathfrak{f}}$ so that we may use the $R$-matrix as in (2.12). We also endow $\widehat{\mathfrak{f}}^{\sigma}$ with a twisted inner product [26]

$$
(X, Y)_{\varphi}=\operatorname{res}_{\lambda=0} \frac{d \lambda}{\lambda} \phi(\lambda)\langle X(\lambda), Y(\lambda)\rangle, \quad \phi(\lambda)=\frac{4 \lambda^{2}}{\left(1-\lambda^{2}\right)^{2}} .
$$

Notice that we have extracted an explicit factor of $\lambda^{-1}$ from the twist function $\varphi(\lambda)=$ $\lambda^{-1} \phi(\lambda)$ so that the remaining twist $\phi(\lambda)$ is invariant under $\lambda \mapsto-\lambda$.

To extract the Poisson brackets of the fundamental fields $A^{(i)}$ and $\Pi^{(i)}$ from (2.8) in this case we consider the following linear functionals

$$
\begin{array}{lll}
a_{\sigma, x}^{(1)}: & \mathcal{L} \mapsto \quad\left(\left(\mathcal{L}, \frac{1}{4}\left(\lambda^{-3}-\lambda^{-1}\right) x^{(1)} \cdot \delta_{\sigma}\right)\right)_{\varphi}=\left\langle A^{(1)}(\sigma), x^{(1)}\right\rangle, \\
\pi_{\sigma, x}^{(1)}: & \mathcal{L} \mapsto \quad\left(\left(\mathcal{L}, \frac{1}{4}\left(3 \lambda^{-1}-\lambda^{-3}\right) x^{(1)} \cdot \delta_{\sigma}\right)\right)_{\varphi}=\left\langle\Pi^{(1)}(\sigma), x^{(1)}\right\rangle, \\
a_{\sigma, x}^{(0)}: & \mathcal{L} \mapsto \quad\left(\left(\mathcal{L}, \frac{1}{4}\left(\lambda^{-4}-\lambda^{-2}\right) x^{(0)} \cdot \delta_{\sigma}\right)\right)_{\varphi}=\left\langle A^{(0)}(\sigma), x^{(0)}\right\rangle, \\
\pi_{\sigma, x}^{(0)}: & \mathcal{L} \mapsto \quad\left(\left(\mathcal{L}, \frac{1}{2}\left(2 \lambda^{-2}-\lambda^{-4}\right) x^{(0)} \cdot \delta_{\sigma}\right)\right)_{\varphi}=\left\langle\Pi^{(0)}(\sigma), x^{(0)}\right\rangle .
\end{array}
$$

In terms of these we can compute for instance,

$$
\begin{aligned}
\left\langle\left\{A_{\underline{\mathbf{1}}}^{(0)}(\sigma), \Pi_{\underline{\mathbf{2}}}^{(0)}\left(\sigma^{\prime}\right)\right\}, x_{\underline{\mathbf{1}}}^{(0)} y_{\underline{\mathbf{2}}}^{(0)}\right\rangle_{\underline{\mathbf{1 2}}} & =\left\{a_{\sigma, x}^{(0)}, \pi_{\sigma^{\prime}, y}^{(0)}\right\}(\mathcal{L}) \\
& =-\left\langle A^{(0)}(\sigma),\left[x^{(0)}, y^{(0)}\right]\right\rangle \delta_{\sigma \sigma^{\prime}}-\left\langle x^{(0)}, y^{(0)}\right\rangle \delta_{\sigma \sigma^{\prime}}^{\prime} .
\end{aligned}
$$

Performing similar calculations, altogether we find exactly the Poisson brackets of the symmetric space $\sigma$-model, namely

$$
\begin{aligned}
& \left\{A_{\underline{\mathbf{1}}}^{(i)}(\sigma), A_{\underline{\mathbf{2}}}^{(j)}\left(\sigma^{\prime}\right)\right\}=0 \\
& \left\{A_{\underline{\mathbf{1}}}^{(i)}(\sigma), \Pi_{\underline{\mathbf{2}}}^{(j)}\left(\sigma^{\prime}\right)\right\}=\left[C_{\underline{\mathbf{1 2}}}^{(i i)}, A_{\underline{\mathbf{2}}}^{(i+j)}(\sigma)\right] \delta_{\sigma \sigma^{\prime}}-\delta_{i j} C_{\underline{\mathbf{1 2}}}^{(i i)} \delta_{\sigma \sigma^{\prime}}^{\prime}, \\
& \left\{\Pi_{\underline{\mathbf{1}}}^{(i)}(\sigma), \underline{\Pi_{\underline{\mathbf{2}}}^{(j)}}\left(\sigma^{\prime}\right)\right\}=\left[C_{\underline{\mathbf{1 2}}}^{(i i)}, \Pi_{\underline{\mathbf{2}}}^{(i+j)}(\sigma)\right] \delta_{\sigma \sigma^{\prime}} .
\end{aligned}
$$

\subsubsection{Mildly non-ultralocal bracket}

Guided by our algebraic reformulation in (2.20) of the Faddeev-Reshetikhin modification of the Poisson bracket, we would like to obtain a similar prescription in the case at hand for symmetric space $\sigma$-model.

Since the $R$-matrix of the model is of the same form as in the principal chiral model case, naively one might try to replace the twisted inner product by the rational inner product also in the present case. However, the latter vanishes identically on $\widehat{f}^{\sigma}$. Indeed, the quantity $\langle X(\lambda) Y(\lambda)\rangle$ in (2.17) is a function of $\lambda^{2}$ and therefore has vanishing residue. We are thus forced to use the simplest non-degenerate inner product on $\widehat{\mathfrak{f}}^{\sigma}$, which is the trigonometric one,

$$
(X, Y)_{\text {trig }}=\operatorname{res}_{\lambda=0} d \lambda \lambda^{-1}\langle X(\lambda), Y(\lambda)\rangle .
$$


This corresponds to the choice of twist function $\varphi^{\prime}(\lambda)=\lambda^{-1}$. So our prescription for modifying the Poisson bracket is simply

$$
\left.\left(\widehat{\mathfrak{f}}^{\sigma}, \mathcal{L}, R, \lambda^{-1} \phi\right) \quad \longrightarrow \quad \widehat{(\mathfrak{f}}^{\sigma}, \mathcal{L}, R, \lambda^{-1}\right) .
$$

In particular, we keep the same $R$-matrix which isn't skew-symmetric with respect to the trigonometric inner product. Therefore the new Poisson bracket is still non-ultralocal! This is in sharp contrast with the Faddeev-Reshetikhin prescription for principal chiral models. However, as we will explain in section 4, it turns out that although the new bracket is still non-ultralocal, it is not plagued with the same problems as the original non-ultralocal bracket. In particular, this better behaved non-ultralocal Poisson structure admits a lattice discretization. We refer to this special type of non-ultralocality as being mild, to be defined more precisely in section 4 . This observation leads to a natural generalization of the Faddeev-Reshetikhin procedure.

To extract the Poisson brackets of the fields $A^{(i)}$ and $\Pi^{(i)}$ we proceed as usual and consider the linear functionals

$$
\begin{array}{llr}
a_{\sigma, x}^{\prime(1)}: & \mathcal{L} \mapsto \quad\left(\left(\mathcal{L},\left(\lambda+\lambda^{-1}\right) x^{(1)} \cdot \delta_{\sigma}\right)\right)_{\text {trig }}=\left\langle A^{(1)}(\sigma), x^{(1)}\right\rangle, \\
\pi_{\sigma, x}^{\prime(1)}: & \mathcal{L} \mapsto \quad\left(\left(\mathcal{L},\left(\lambda-\lambda^{-1}\right) x^{(1)} \cdot \delta_{\sigma}\right)\right)_{\text {trig }}=\left\langle\Pi^{(1)}(\sigma), x^{(1)}\right\rangle, \\
a_{\sigma, x}^{\prime(0)}: & \mathcal{L} \mapsto \quad\left(\left(\mathcal{L},\left(1+\lambda^{-2}\right) x^{(0)} \cdot \delta_{\sigma}\right)\right)_{\text {trig }}=\left\langle A^{(0)}(\sigma), x^{(0)}\right\rangle, \\
\pi_{\sigma, x}^{\prime(0)}: & \mathcal{L} \mapsto \quad\left(\left(\mathcal{L},-2 \lambda^{-2} x^{(0)} \cdot \delta_{\sigma}\right)\right)_{\text {trig }}=\left\langle\Pi^{(0)}(\sigma), x^{(0)}\right\rangle .
\end{array}
$$

Using these expressions we can explicitly compute the Poisson brackets between the various fields $A^{(i)}$ and $\Pi^{(i)}$. For instance,

$$
\begin{aligned}
\left\langle\left\{A_{\underline{\mathbf{1}}}^{(0)}(\sigma), A_{\underline{\mathbf{2}}}^{(0)}\left(\sigma^{\prime}\right)\right\}^{\prime}, x_{\underline{\mathbf{1}}}^{(0)} y_{\underline{\mathbf{2}}}^{(0)}\right\rangle_{\underline{\mathbf{1 2}}} & =\left\{a_{\sigma, x}^{\prime(0)}, a_{\sigma^{\prime}, y}^{\prime(0)}\right\}^{\prime}(\mathcal{L}) \\
& =\left\langle 2 A^{(0)}(\sigma)+\Pi^{(0)}(\sigma),\left[x^{(0)}, y^{(0)}\right]\right\rangle \delta_{\sigma \sigma^{\prime}}+2\left\langle x^{(0)}, y^{(0)}\right\rangle \delta_{\sigma \sigma^{\prime}}^{\prime} .
\end{aligned}
$$

Similarly all the other Poisson brackets can be computed. The final result for all the brackets reads

$$
\begin{aligned}
& \left\{A_{\underline{\mathbf{1}}}^{(0)}(\sigma), A_{\underline{\mathbf{2}}}^{(0)}\left(\sigma^{\prime}\right)\right\}^{\prime}=-\left[C_{\underline{\mathbf{1 2}}}^{(00)}, 2 A_{\underline{\mathbf{2}}}^{(0)}(\sigma)+\Pi_{\underline{\mathbf{2}}}^{(0)}(\sigma)\right] \delta_{\sigma \sigma^{\prime}}+2 C_{\underline{\mathbf{1 2}}}^{(00)} \delta_{\sigma \sigma^{\prime}}^{\prime}, \\
& \left\{A_{\underline{\mathbf{1}}}^{(0)}(\sigma), A_{\underline{\mathbf{2}}}^{(1)}\left(\sigma^{\prime}\right)\right\}^{\prime}=-\left[C_{\underline{\mathbf{1 2}}}^{(00)}, A_{\underline{\mathbf{2}}}^{(1)}(\sigma)+\Pi_{\underline{\mathbf{2}}}^{(1)}(\sigma)\right] \delta_{\sigma \sigma^{\prime}}, \\
& \left\{A_{\underline{\mathbf{1}}}^{(0)}(\sigma), \Pi_{\underline{\mathbf{2}}}^{(0)}\left(\sigma^{\prime}\right)\right\}^{\prime}=0, \\
& \left\{A_{\underline{\mathbf{1}}}^{(0)}(\sigma), \Pi_{\underline{\mathbf{2}}}^{(1)}\left(\sigma^{\prime}\right)\right\}^{\prime}=-\left[C_{\underline{\underline{\mathbf{2}}}}^{(00)}, A_{\underline{\mathbf{2}}}^{(1)}(\sigma)+\Pi_{\underline{\mathbf{2}}}^{(1)}(\sigma)\right] \delta_{\sigma \sigma^{\prime}}, \\
& \left\{A_{\underline{\mathbf{1}}}^{(1)}(\sigma), A_{\underline{\mathbf{2}}}^{(1)}\left(\sigma^{\prime}\right)\right\}^{\prime}=-\left[C_{\underline{\mathbf{1 2}}}^{(11)}, \Pi_{\underline{\mathbf{2}}}^{(0)}(\sigma)\right] \delta_{\sigma \sigma^{\prime}}, \\
& \left\{A_{\underline{\mathbf{1}}}^{(1)}(\sigma), \Pi_{\underline{\mathbf{2}}}^{(0)}\left(\sigma^{\prime}\right)\right\}^{\prime}=0, \\
& \left\{A_{\underline{\mathbf{1}}}^{(1)}(\sigma), \Pi_{\underline{\mathbf{2}}}^{(1)}\left(\sigma^{\prime}\right)\right\}^{\prime}=\left[C_{\underline{\mathbf{1 2}}}^{(11)}, \Pi_{\underline{\mathbf{2}}}^{(0)}(\sigma)\right] \delta_{\sigma \sigma^{\prime},} \\
& \left\{\Pi_{\underline{\mathbf{1}}}^{(0)}(\sigma), \Pi_{\underline{\mathbf{2}}}^{(0)}\left(\sigma^{\prime}\right)\right\}^{\prime}=0, \\
& \left\{\Pi_{\underline{\mathbf{1}}}^{(0)}(\sigma), \Pi_{\underline{\mathbf{2}}}^{(1)}\left(\sigma^{\prime}\right)\right\}^{\prime}=0, \\
& \left\{\Pi_{\underline{\mathbf{1}}}^{(1)}(\sigma), \Pi_{\underline{\mathbf{2}}}^{(1)}\left(\sigma^{\prime}\right)\right\}^{\prime}=-\left[C_{\underline{\mathbf{1 2}}}^{(11)}, \Pi_{\underline{\mathbf{2}}}^{(0)}(\sigma)\right] \delta_{\sigma \sigma^{\prime}} .
\end{aligned}
$$

Note that only the Poisson bracket of the field $A^{(0)}$ with itself is non-ultralocal. 


\subsection{Compatibility of the Poisson brackets}

It turns out that for both the principal chiral model and symmetric space $\sigma$-model considered above, the new Poisson bracket $\{\cdot, \cdot\}^{\prime}$ is compatible with the original one $\{\cdot, \cdot\}$, in the sense that any linear combination $u\{\cdot, \cdot\}^{\prime}+v\{\cdot, \cdot\}$ is also a Poisson bracket.

To explain the origin of this property, we restrict ourselves to the case of the principal chiral model. We have shown that the original Poisson bracket is associated with the data $\widehat{(\mathfrak{f}}, \mathcal{L}, R, \varphi)$, with $\varphi$ given by (2.13), through the formula (2.8). However, an equivalent set of data producing the same bracket through $(2.8)$ is $\left(\widehat{\mathfrak{f}}, \mathcal{L}, R \circ \tilde{\varphi}^{-1}, 1\right)$. Indeed, the Poisson bracket (2.8) can be rewritten purely in terms of the rational inner product and corresponding cocycle provided we use the twisted $R$-matrix $R_{\varphi^{-1}}=R \circ \tilde{\varphi}^{-1}$ instead of $R$. Note that $R_{\varphi^{-1}}$ also satisfies the $\operatorname{mCYBE}(2.4)$ but with $\omega=\varphi^{-2}$. Explicitly we have

$$
\begin{aligned}
\{f, g\}(\mathcal{L})= & \left(\left(\mathcal{L},\left[R_{\varphi^{-1}} d_{1} f, d_{1} g\right]+\left[d_{1} f, R_{\varphi^{-1}} d_{1} g\right]\right)\right)_{\mathrm{rat}} \\
& +\left(\omega_{\mathrm{rat}}\left(R_{\varphi^{-1}} d_{1} f, d_{1} g\right)+\omega_{\mathrm{rat}}\left(d_{1} f, R_{\varphi^{-1}} d_{1} g\right)\right) .
\end{aligned}
$$

Comparing the Poisson bracket of the principal chiral model in this form to the FaddeevReshetikhin one given by (2.18), we see that the Faddeev-Reshetikhin procedure (2.20) equivalently reads

$$
\left.\left.\widehat{(\mathfrak{f}}, \mathcal{L}, R_{\varphi^{-1}}, 1\right) \quad \longrightarrow \quad \widehat{(\mathfrak{f}}, \mathcal{L}, R, 1\right) .
$$

Now it is well known [27] that given an $R$-matrix $R$, for any $q \in \mathbb{C}((\lambda))$ the $R$-bracket associated with $R$ and $R \circ \tilde{q}$ are compatible. Therefore, taking $q=\varphi^{-1}$ in the present case we conclude that the Poisson brackets (2.8) and (2.18) are compatible.

Likewise, in the case of a symmetric space $\sigma$-model, the generalized FaddeevReshetikhin procedure can be understood as

$$
\left.\left(\widehat{\mathfrak{f}}^{\sigma}, \mathcal{L}, R, \lambda^{-1} \phi\right) \sim\left(\widehat{\mathfrak{f}}^{\sigma}, \mathcal{L}, R_{\phi^{-1}}, \lambda^{-1}\right) \quad \longrightarrow \quad \widehat{\mathfrak{f}}^{\sigma}, \mathcal{L}, R, \lambda^{-1}\right) .
$$

Note that in the case of a twisted loop algebra such as $\widehat{\mathfrak{f}}^{\sigma}$, the conclusion about compatibility of the $R$-brackets associated with $R$ and $R \circ \tilde{q}$ remains valid provided we use a $q \in \mathbb{C}((\lambda))$ such that $q(\lambda)=q(-\lambda)$. In particular we may take $q=\phi^{-1}$, from which the desired compatibility follows.

\section{Dynamics and Hamiltonian}

Having made the choice of the Poisson bracket (2.24), and following the method used by Faddeev and Reshetikhin as given in [6], we now study the dynamics of the symmetric space $\sigma$-model on $F / G$ where $G$ is the Lie group corresponding to $\mathfrak{g}=\mathfrak{f}^{(0)}$ and determine which reduction of the field equations may be obtained in a Hamiltonian framework.

\subsection{Original dynamics and gauge invariance}

Let us start by recalling the expression for the Hamiltonian of the $F / G$ coset $\sigma$-model. The theory is conformally invariant at the classical level and the components of the stress-energy 
tensor $\operatorname{are}^{3}$

$$
T_{ \pm \pm}=-\frac{1}{4} \operatorname{Tr}\left(A_{ \pm}^{(1)} A_{ \pm}^{(1)}\right)
$$

where $A_{ \pm}^{(1)}=\Pi^{(1)} \mp A^{(1)}$. In terms of these the Hamiltonian reads

$$
H=\int d \sigma\left(T_{++}+T_{--}+\operatorname{Tr}\left(A^{(0)} \Pi^{(0)}\right)+\operatorname{Tr}\left(\ell \Pi^{(0)}\right)\right) .
$$

The field $\Pi^{(0)}$ is the constraint associated with the coset gauge invariance. Its Lagrange multiplier $\ell$ takes values in $\mathfrak{g}$. The term $\operatorname{Tr}\left(A^{(0)} \Pi^{(0)}\right)$ has been taken into account in order to be consistent with the analysis of [25] on the Hamiltonian Lax connection. By construction, the constraint $\Pi^{(0)}$ is preserved by the dynamics generated by the Hamiltonian $H$. The equations of motion for the other variables $\left(A^{(0)}, A_{ \pm}^{(1)}\right)$ are, up to terms proportional to the constraint $\Pi^{(0)}$,

$$
\begin{aligned}
& \partial_{-} A^{(0)}=-\frac{1}{2}\left[A_{+}^{(1)}, A_{-}^{(1)}\right]+\partial_{\sigma} \ell+\left[\ell, A^{(0)}\right], \\
& \partial_{-} A_{+}^{(1)}=\left[\ell, A_{+}^{(1)}\right], \\
& \partial_{+} A_{-}^{(1)}=-\left[A_{-}^{(1)}, 2 A^{(0)}+\ell\right]
\end{aligned}
$$

where $\partial_{ \pm}=\partial_{\tau} \pm \partial_{\sigma}$. The fact that the dynamics depends on the arbitrary function $\ell$ of $\sigma$ and $\tau$ is a reflection of the gauge invariance generated by the constraint $\Pi^{(0)}$. The corresponding gauge transformation of the fields reads

$$
\begin{aligned}
\delta A^{(0)} & =\left[\alpha_{R}, A^{(0)}\right]+\partial_{\sigma} \alpha_{R} \\
\delta A_{ \pm}^{(1)} & =\left[\alpha_{R}, A_{ \pm}^{(1)}\right] \\
\delta \ell & =\partial_{-} \alpha_{R}+\left[\alpha_{R}, \ell\right]
\end{aligned}
$$

with $\alpha_{R}$ a function taking values in $\mathfrak{g}$. The index $R$ is used to emphasize that we are considering a right coset $F / G$. Note that a direct consequence of the equations of motion is that

$$
\partial_{\mp} \operatorname{Tr}\left[\left(A_{ \pm}^{(1)}\right)^{n}\right]=0 .
$$

\subsection{Casimirs of the new Poisson bracket and Pohlmeyer reduction}

Before attempting to reproduce the above dynamics in terms of the modified Poisson bracket (2.24), we first need to identify the Casimirs of the latter. Indeed, since these quantities will necessarily remain constant in time with respect to (2.24), we shall only be able to reproduce a reduction of the original dynamics where the same quantities have been set to constants.

The field $\Pi^{(0)}$ is an obvious Casimir of (2.24). Since it is a constraint of the symmetric space $\sigma$-model, it is natural to set the value of this Casimir to zero. Next, we have

$$
\begin{array}{ll}
\left\{A_{\underline{\mathbf{1}}}^{(0)}(\sigma), A_{-\underline{\mathbf{2}}}^{(1)}\left(\sigma^{\prime}\right)\right\}^{\prime}=-2\left[C_{\underline{\mathbf{1 2}}}^{(00)}, A_{-\underline{\mathbf{2}}}^{(1)}(\sigma)\right] \delta_{\sigma \sigma^{\prime}}, & \left\{A_{\underline{\mathbf{1}}}^{(1)}(\sigma), A_{-\underline{\mathbf{2}}}^{(1)}\left(\sigma^{\prime}\right)\right\}^{\prime}=0, \\
\left\{\Pi_{\underline{\mathbf{1}}}^{(0)}(\sigma), A_{-\underline{\mathbf{2}}}^{(1)}\left(\sigma^{\prime}\right)\right\}^{\prime}=0, & \left\{\Pi_{\underline{\mathbf{1}}}^{(1)}(\sigma), A_{-\underline{\mathbf{2}}}^{(1)}\left(\sigma^{\prime}\right)\right\}^{\prime}=0 .
\end{array}
$$

\footnotetext{
${ }^{3}$ We have taken the relation between the inner product and the trace of the product in some representation to be $\langle A, B\rangle=-\operatorname{Tr}(A B)$. This leads to a positive inner product when $F$ is compact.
} 
This implies that the quantities $\operatorname{Tr}\left[\left(A_{-}^{(1)}\right)^{n}\right]$ are also Casimirs of this Poisson bracket. The existence of these other Casimirs is the first sign that one will have to perform a Pohlmeyer reduction of the coset $\sigma$-model. Equation (3.3) shows that the densities $\operatorname{Tr}\left[\left(A_{-}^{(1)}\right)^{n}\right]$ are chiral in the original model and may therefore be set to constants. However, in order to do this reduction in a consistent manner, one must take into consideration the fact that these quantities are not all independent (see for instance $[28,29]$ ). We follow the references $[18,19]$. The number of independent quantities corresponds to the rank of $F / G$, which is defined to be the dimension of the maximal abelian subspaces of $\mathfrak{f}^{(1)}$. For instance, in the case of $S^{n}$ or $A d S_{n}$ this dimension is one. This is however not the end of the story as there is a further simplification. Indeed, when $\Pi^{(0)}=0$, the only non-vanishing Poisson brackets in (2.24) are

$$
\begin{aligned}
& \left\{A_{\underline{\mathbf{1}}}^{(0)}(\sigma), A_{\underline{\mathbf{2}}}^{(0)}\left(\sigma^{\prime}\right)\right\}^{\prime}=-2\left[C_{\underline{\mathbf{1 2}}}^{(00)}, A_{\underline{\mathbf{2}}}^{(0)}(\sigma)\right] \delta_{\sigma \sigma^{\prime}}+2 C_{\underline{\mathbf{1 2}}}^{(00)} \delta_{\sigma \sigma^{\prime}}^{\prime}, \\
& \left\{A_{\underline{\mathbf{1}}}^{(0)}(\sigma), A_{-\underline{\mathbf{2}}}^{(1)}\left(\sigma^{\prime}\right)\right\}^{\prime}=-2\left[C_{\underline{\mathbf{1 2}}}^{(00)}, A_{-\underline{\mathbf{2}}}^{(1)}(\sigma)\right] \delta_{\sigma \sigma} .
\end{aligned}
$$

This implies, in particular, that $A_{+}^{(1)}$ is yet another Casimir. At this stage it is clear that we face exactly the same situation as in the Pohlmeyer reduction. We shall therefore fix the value of each Casimir to coincide with the value of the same quantity in the Pohlmeyer reduced coset $\sigma$-model.

Let $\mathfrak{a}$ be a maximal abelian subspace of $\mathfrak{f}^{(1)}$. We fix the value of the Casimir $A_{+}^{(1)}$ by setting

$$
A_{+}^{(1)}=\mu_{+} T_{+},
$$

where $T_{+} \in \mathfrak{a}$ and $\mu_{+} \in \mathbb{R}$ are constant. ${ }^{4}$ Within the scheme of the Pohlmeyer reduction, condition (3.5) is the result of two separate steps (see for instance $[18,19]$ ). The first one corresponds to imposing the partial gauge fixing condition $A_{+}^{(1)}(\sigma, \tau)=\mu_{+}(\sigma, \tau) T_{+}$. The fact that this is a valid partial gauge fixing condition follows from the polar decomposition theorem. ${ }^{5}$ The second step consists in fixing the on-shell chiral function $\mu_{+}(\sigma, \tau)$ to a constant using a holomorphic conformal transformation, which corresponds to a partial reduction of conformal symmetry.

The adjoint action $\operatorname{Ad}_{T_{+}}$of the element $T_{+} \in \mathfrak{a}$ defines a decomposition of $\mathfrak{f}$. We shall denote it as $\mathfrak{f}=\mathfrak{f}^{[0]} \oplus \mathfrak{f}^{[1]}$ where

$$
\mathfrak{f}^{[0]}=\operatorname{Ker}\left(\operatorname{Ad}_{T_{+}}\right) \quad \text { and } \quad \mathfrak{f}^{[1]}=\operatorname{Im}\left(\operatorname{Ad}_{T_{+}}\right),
$$

which satisfy $\left[\mathfrak{f}^{[0]}, \mathfrak{f}^{[0]}\right] \subset \mathfrak{f}^{[0]}$ and $\left[\mathfrak{f}^{[0]}, \mathfrak{f}^{[1]}\right] \subset \mathfrak{f}^{[1]}$. We define $\mathfrak{h}_{R}=\mathfrak{g}^{[0]}$ and let $H_{R} \subset G$ be the corresponding Lie subgroup which consists of elements commuting with $T_{+}$. For instance, in the case of $S^{n}=\mathrm{SO}(n+1) / \mathrm{SO}(n)$ we have $H_{R} \simeq \mathrm{SO}(n-1)$. We immediately see from $(3.1 \mathrm{~b})$ that the stability of condition $(3.5)$ under time evolution requires $\left[T_{+}, \ell\right]=0$, i.e. $\ell \in \mathfrak{h}_{R}$ or equivalently $\ell=\ell^{[0]}$. Likewise, it then follows from (3.2) that the residual gauge transformations preserving (3.5) are such that $\alpha_{R} \in \mathfrak{h}_{R}$.

\footnotetext{
${ }^{4}$ In the case of $A d S_{n}$, it is possible to choose a $T_{+}$that would correspond to the vanishing of the components $T_{++}$of the stress-energy tensor. We do not consider this case here.

${ }^{5}$ The polar decomposition theorem is only valid when the group $G$ is compact. An extension of this theorem to the case of anti-de Sitter spaces may be found for instance in [19].
} 
Next, we pick another element $T_{-} \in \mathfrak{a}$. As for $T_{+}$, this element defines its own decomposition of $\mathfrak{f}$ along with a Lie algebra $\mathfrak{h}_{L}=\mathfrak{g} \cap \operatorname{Ker}\left(A d_{T_{-}}\right)$and its corresponding Lie subgroup $H_{L} \subset G$ of elements commuting with $T_{-}$. Sticking to our general strategy we have to fix the values of all the Casimirs $\operatorname{Tr}\left[\left(A_{-}^{(1)}\right)^{n}\right]$. Introducing a new field $g$ taking value in $G$ through the use of the polar decomposition theorem, this may be done by setting

$$
A_{-}^{(1)}=\mu_{-} g^{-1} T_{-} g
$$

From the point of view taken in this article, the fact that $A_{-}^{(1)}$ is related by the adjoint action of $g$ to a constant matrix $\mu_{-} T_{-}$comes from the necessity to fix the Casimirs $\operatorname{Tr}\left[\left(A_{-}^{(1)}\right)^{n}\right]$. From the point of view of the Pohlmeyer reduction, the equation (3.6) is reached by using conformal invariance, or an extension of conformal invariance in the case where the dimension of $\mathfrak{a}$ is bigger than 1. In both cases, however, consistency of (3.6) with the dynamics is ensured by the fact that the Casimirs $\operatorname{Tr}\left[\left(A_{-}^{(1)}\right)^{n}\right]$ are chiral densities. However, $g$ is clearly not uniquely defined by (3.6). Indeed, the transformation

$$
g \rightarrow h_{L} g
$$

where $h_{L}$ is a $H_{L}$-valued function, leads to the same $A_{-}^{(1)}$.

In the following we shall restrict to the case where there exists an automorphism $\iota$ of the algebra $\mathfrak{f}$ which relates $T_{-}$and $T_{+}$as $T_{+}=\iota\left(T_{-}\right)$, and with the following properties

$$
\iota\left(\mathfrak{f}^{(i)}\right) \subset \mathfrak{f}^{(i)}, i=0,1 \quad \text { and } \quad \operatorname{Tr}(\iota(M) \iota(N))=\operatorname{Tr}(M N), M, N \in \mathfrak{f} .
$$

In particular, the automorphism $\iota$ relates the right algebra $\mathfrak{h}_{R}$ with the left algebra $\mathfrak{h}_{L}$, $\mathfrak{h}_{R}=\iota\left(\mathfrak{h}_{L}\right)$.

\subsection{Lifting to $G$}

\subsubsection{Poisson brackets}

At this point the phase space is parametrized by $g$ and $A^{(0)}$ taking values in $G$ and $\mathfrak{g}$ respectively. We therefore need to lift the Poisson brackets (3.4) to the pair of fields $\left(A^{(0)}, g\right)$. To avoid clutter, in the rest of this section we suppress superscripts corresponding to the $\mathbb{Z}_{2}$-grading, i.e. we write $A \equiv A^{(0)}, A_{-} \equiv A_{-}^{(1)}$ and

$$
\begin{aligned}
\left.\left\{A_{-\underline{\mathbf{1}}}(\sigma), A_{-\underline{\mathbf{2}}}\left(\sigma^{\prime}\right)\right)\right\}^{\prime} & =0 \\
\left\{A_{-\underline{\mathbf{1}}}(\sigma), A_{\underline{\mathbf{2}}}\left(\sigma^{\prime}\right)\right\}^{\prime} & =2\left[C_{\underline{\mathbf{1 2}}}^{(00)}, A_{-\underline{\mathbf{1}}}(\sigma)\right] \delta_{\sigma \sigma^{\prime}} .
\end{aligned}
$$

The first Poisson bracket (3.9a) is clearly satisfied if we let $\left\{g(\sigma), g\left(\sigma^{\prime}\right)\right\}^{\prime}=0$. Consider now the second Poisson bracket (3.9b). Using the relation, following from (3.6),

$$
\left\{A_{-\underline{\mathbf{1}}}(\sigma), A_{\underline{\mathbf{2}}}\left(\sigma^{\prime}\right)\right\}^{\prime}=\left[A_{-\underline{\mathbf{1}}}(\sigma), g_{\underline{\mathbf{1}}}(\sigma)^{-1}\left\{g_{\underline{\mathbf{1}}}(\sigma), A_{\underline{\mathbf{2}}}\left(\sigma^{\prime}\right)\right\}^{\prime}\right]
$$

we observe that $(3.9 \mathrm{~b})$ is satisfied if we introduce the following Poisson bracket

$$
\left\{g_{\underline{\mathbf{1}}}(\sigma), A_{\underline{\mathbf{2}}}\left(\sigma^{\prime}\right)\right\}^{\prime}=-2 g_{\underline{\mathbf{1}}}(\sigma) C_{\underline{\mathbf{1 2}}}^{(00)} \delta_{\sigma \sigma^{\prime}} .
$$


The complete set of Poisson brackets between the fields $g$ and $A$ therefore reads

$$
\begin{aligned}
&\left\{g_{\underline{\mathbf{1}}}(\sigma), g_{\underline{\mathbf{2}}}\left(\sigma^{\prime}\right)\right\}^{\prime}=0, \\
&\left\{g_{\underline{\mathbf{1}}}(\sigma), A_{\underline{\mathbf{2}}}\left(\sigma^{\prime}\right)\right\}^{\prime}=-2 g_{\underline{\mathbf{1}}}(\sigma) C_{\underline{\mathbf{1 2}}}^{(00)} \delta_{\sigma \sigma^{\prime}}, \\
&\left\{A_{\underline{\mathbf{1}}}(\sigma), A_{\underline{\mathbf{2}}}\left(\sigma^{\prime}\right)\right\}^{\prime}=-2\left[C_{\underline{\mathbf{1 2}}}^{(00)}, A_{\underline{\mathbf{2}}}(\sigma)\right] \delta_{\sigma \sigma^{\prime}}+2 C_{\underline{\mathbf{1 2}}}^{(00)} \delta_{\sigma \sigma^{\prime}}^{\prime} .
\end{aligned}
$$

This is precisely the canonical Poisson brackets of the WZW model with group $G$ (see e.g. [20]).

\subsubsection{Reduced original dynamics}

The equations (3.1a) and (3.1c) that remain after imposing (3.5) and (3.6) are

$$
\begin{aligned}
\partial_{-} A^{[0]} & =\partial_{\sigma} \ell+\left[\ell, A^{[0]}\right], \\
\partial_{-} A^{[1]} & =-\frac{1}{2} \mu_{+} \mu_{-}\left[T_{+}, g^{-1} T_{-} g\right]+\left[\ell, A^{[1]}\right], \\
\partial_{+} A_{-} & =-\left[A_{-}, 2 A+\ell\right] .
\end{aligned}
$$

Here we have extracted the components of (3.1a) along $\mathfrak{f}^{[0]}$ and $\mathfrak{f}^{[1]}$ using the properties of these spaces and the fact that $\ell \in \mathfrak{h}_{R}$. These equations of motion are invariant under the infinitesimal gauge transformations

$$
\begin{aligned}
\delta A^{[0]} & =\left[\alpha_{R}, A^{[0]}\right]+\partial_{\sigma} \alpha_{R}, \\
\delta A^{[1]} & =\left[\alpha_{R}, A^{[1]}\right] \\
\delta \ell & =\partial_{-} \alpha_{R}+\left[\alpha_{R}, \ell\right], \\
\delta g & =\alpha_{L} g-g \alpha_{R} \Longrightarrow \delta A_{-}=\left[\alpha_{R}, A_{-}\right]
\end{aligned}
$$

where the functions $\alpha_{R}$ and $\alpha_{L}$ take values in $\mathfrak{h}_{R}$ and $\mathfrak{h}_{L}$ respectively. Next, we lift the equation of motion (3.12c) to an equation of motion for $g$. Using the property $\partial_{+} A_{-}=$ $\left[A_{-}, g^{-1} \partial_{+} g\right]$, we find

$$
\left[T_{-}, \partial_{+} g g^{-1}+g(2 A+\ell) g^{-1}\right]=0 \text {. }
$$

This shows that the equation of motion for $g$ is

$$
\partial_{+} g g^{-1}+g(2 A+\ell) g^{-1}=\tilde{\ell}
$$

where $\tilde{\ell}$ is an arbitrary function taking value in the subalgebra $\mathfrak{h}_{L} \subset \mathfrak{g}$. Its presence reflects the appearance of the left gauge invariance (3.7) upon introducing the phase space field $g$ to replace $A_{-}$. The equation (3.14) is invariant under the complete set of gauge transformations (3.13) provided that the function $\tilde{\ell}$ transforms as

$$
\delta \tilde{\ell}=\partial_{+} \alpha_{L}+\left[\alpha_{L}, \widetilde{\ell}\right] .
$$

In other words, $\widetilde{\ell}$ behaves as a gauge field for the gauge invariance under the left group $H_{L}$. For later use, let us write down the expression of $A$ coming from equation (3.14)

$$
A=\frac{1}{2}\left(g^{-1} \tilde{\ell} g-g^{-1} \partial_{+} g-\ell\right) .
$$

We shall need, in particular, the projection of this equation to $\mathfrak{h}_{R}$

$$
A^{[0]}=\frac{1}{2}\left(-\ell+\left(g^{-1} \tilde{\ell} g-g^{-1} \partial_{+} g\right)^{[0]}\right) .
$$




\subsubsection{Gauge invariances and anomalies}

We are now ready to look for a functional which generates the infinitesimal gauge transformations (3.13) on the phase space fields $g$ and $A$ in terms of the Poisson bracket (3.11). This is easily found to be

$$
\frac{1}{2} \int d \sigma \operatorname{Tr}\left(\alpha_{L} J-A \alpha_{R}\right)
$$

where the current $J$ is defined by

$$
J=\partial_{\sigma} g g^{-1}+g A g^{-1} .
$$

Indeed, a simple computation leads to the following Poisson brackets

$$
\begin{aligned}
&\left\{J_{\underline{\mathbf{1}}}(\sigma), g_{\underline{\mathbf{2}}}\left(\sigma^{\prime}\right)\right\}^{\prime}=2 C_{\underline{\mathbf{1 2}}}^{(00)} g_{\underline{\mathbf{2}}}(\sigma) \delta_{\sigma \sigma^{\prime}}, \\
&\left\{J_{\underline{\mathbf{1}}}(\sigma), A_{\underline{\mathbf{2}}}\left(\sigma^{\prime}\right)\right\}^{\prime}=0 .
\end{aligned}
$$

The second equation means that gauge transformations with parameter $\alpha_{L}$ do not act on the field $A$, as it should be, but only generate a left multiplication on $g$. Furthermore, the current $J$ satisfies

$$
\left\{J_{\underline{\mathbf{1}}}(\sigma), J_{\underline{\mathbf{2}}}\left(\sigma^{\prime}\right)\right\}^{\prime}=2\left[C_{\underline{\mathbf{1 2}}}^{(00)}, J_{\underline{\mathbf{2}}}(\sigma)\right] \delta_{\sigma \sigma^{\prime}}-2 C_{\underline{\mathbf{1 2}}}^{(00)} \delta_{\sigma \sigma^{\prime}}^{\prime} .
$$

At this stage we have field equations which possess a gauge invariance with gauge group $H_{L} \times H_{R}$, the infinitesimal gauge transformations of which are respectively generated by the field $J$ restricted to $\mathfrak{h}_{L}$ and the field $A$ restricted to $\mathfrak{h}_{R}$. However, neither of these generators have first class Poisson brackets, as is apparent from (3.20) and (3.11c). This is just a reflection of the well-known fact that left and right isometries of the Wess-Zumino-Witten model cannot be freely gauged [30]. However, we also know that a diagonal subgroup of left and right isometries may be gauged. In our case, we indeed find that the combination $\iota(J)-A$ of the left and right generators has first class Poisson brackets. Using (3.20) and (3.11c) along with the properties (3.8) of the automorphism $\iota$, we get

$$
\left\{\iota\left(J_{\underline{\mathbf{1}}}(\sigma)\right)-A_{\underline{\mathbf{1}}}(\sigma), \iota\left(J_{\underline{\mathbf{2}}}\left(\sigma^{\prime}\right)\right)-A_{\underline{\mathbf{2}}}\left(\sigma^{\prime}\right)\right\}^{\prime}=2\left[C_{\underline{\mathbf{1 2}}}^{(00)}, \iota\left(J_{\underline{\mathbf{2}}}(\sigma)\right)-A_{\underline{\mathbf{2}}}(\sigma)\right] \delta_{\sigma \sigma^{\prime}} .
$$

Before looking for a Hamiltonian formulation of the field equations (3.12a), (3.12b) and (3.14), we will thus be led to partially fix the gauge invariance. In this process we shall need the following expression for the part of $\iota(J)$ lying in $\mathfrak{h}_{R}$, which is easily established using the field equation (3.14) and the definition (3.19),

$$
\iota(J)^{[0]}=\frac{1}{2}\left(\iota(\widetilde{\ell})-\iota\left(g \ell g^{-1}-\partial_{-} g g^{-1}\right)^{[0]}\right) .
$$

\subsection{New Hamiltonian}

\subsubsection{Partial gauge fixing on equations of motion}

A short calculation shows that the field $J$ transforms under the gauge transformations (3.13) as

$$
\delta J=\partial_{\sigma} \alpha_{L}+\left[\alpha_{L}, J\right]
$$


We are going to fix the part of the gauge invariance characterized by the relation $\iota\left(\alpha_{L}\right)=-\alpha_{R}$. Under such gauge transformations, using (3.13a) and (3.23), we find the transformation

$$
\delta(\iota(J)-A)=-2 \partial_{\sigma} \alpha_{R}-\left[\alpha_{R},(\iota(J)+A)\right]
$$

We take as partial gauge condition

$$
(\iota(J)-A)^{[0]}=0
$$

This leaves a gauge invariance where the left and right transformations are now related by

$$
\iota\left(\alpha_{L}\right)=\alpha_{R}
$$

Let us note that the equation (3.24) comes out as a partial gauge fixing condition in our study of the field equations, and that it will also play the role of a constraint generating the remaining gauge invariance in the Hamiltonian framework that will soon be described. The last step before determining the Hamiltonian is to work out the consequences of the constraint (3.24). Starting from the definition (3.19) of $J$ and using the equations of motion (3.12a), (3.12b) and (3.14) for $A$ and $g$ we derive the equation of motion for $J$ to be

$$
\partial_{+} J=\partial_{\sigma} \tilde{\ell}+[\tilde{\ell}, J]-\frac{1}{2} \mu_{+} \mu_{-}\left[g T_{+} g^{-1}, T_{-}\right]
$$

Combining this with the equation of motion (3.12a) for $A^{[0]}$, we find that the constraint (3.24) is preserved by the dynamics if

$$
\iota(\widetilde{\ell})=\ell+2 \iota(J)^{[0]} .
$$

Using the equations (3.17) and (3.22), the gauge constraint (3.24) and its dynamical consequence (3.25) may equivalently be written as the set of two equations

$$
\begin{aligned}
\ell & =\iota\left(g \ell g^{-1}+\partial_{-} g g^{-1}\right)^{[0]} \\
\iota(\widetilde{\ell}) & =\left(g^{-1} \widetilde{\ell} g-g^{-1} \partial_{+} g\right)^{[0]}
\end{aligned}
$$

Finally, using the expression (3.16) for the field $A$ enables us to rewrite the second order field equations (3.12a) and (3.12b) as

$$
\left[\partial_{-}-\ell, \partial_{+}+g^{-1} \partial_{+} g-g^{-1} \tilde{\ell} g\right]=\mu_{+} \mu_{-}\left[T_{+}, g^{-1} T_{-} g\right] .
$$

The equations of motion (3.26) coincide with the equations of motion of a $G / H$ gauged WZW model with a potential, where the asymmetric coset $G / H$ is defined as

$$
G / H=G /\left[g \sim h_{L} g \iota\left(h_{L}^{-1}\right) \mid h_{L} \in H_{L}\right] .
$$

Making the comparison for instance with [19], we see agreement with equations (3.40)(3.41) there provided we identify the variables $\mathcal{A}_{ \pm}$there as $\mathcal{A}_{+}=-\widetilde{\ell}$ and $\mathcal{A}_{-}=-\ell$. 


\subsubsection{Hamiltonian}

At last we are ready to describe the above dynamics on the phase space parametrized by the fields $A$ and $g$ with respect to the Poisson bracket (3.11), taking into account the first class constraint (3.24). It is easy to check that the Hamiltonian is given by

$H^{\prime}=\int d \sigma \operatorname{Tr}\left(-\frac{1}{4} \iota(J)^{[1]} \iota(J)^{[1]}-\frac{1}{4} A^{[1]} A^{[1]}+\frac{1}{2}\left(\ell+A^{[0]}\right)\left(\iota(J)^{[0]}-A^{[0]}\right)+\frac{1}{4} \mu_{+} \mu_{-} g^{-1} T_{-} g T_{+}\right)$.

Indeed, its Poisson brackets with $g$ and $A$ are, up to terms proportional to the constraint (3.24),

$$
\begin{aligned}
\left\{H^{\prime}, g\right\}^{\prime} & =\widetilde{\ell} g-g \ell-J g-g A, \\
\left\{H^{\prime}, A\right\}^{\prime} & =\partial_{\sigma} A+\partial_{\sigma} \ell+[\ell, A]-\frac{1}{2} \mu_{+} \mu_{-}\left[T_{+}, g^{-1} T_{-} g\right] .
\end{aligned}
$$

Here we have introduced $\tilde{\ell}$ through the equation $\iota(\widetilde{\ell})=\ell+2 A^{[0]}$ which is equivalent to $(3.25)$ and made use of the properties (3.8) of the automorphism $\iota$. The first equation gives the Hamiltonian form of (3.14) after substituting the definition (3.19) of $J$, and the second equation is equivalent to the Hamiltonian form of (3.12a) and (3.12b). Let us finally note that the Hamiltonian (3.27) is consistent with the result (3.23) of [20].

\subsubsection{Lax pair}

Recall from section 2 that by definition the generalized Faddeev-Reshetikhin model has the same Lax matrix $\mathcal{L}(\lambda)$ as the original theory. In its expression (2.21) for coset $\sigma$-models, we can of course replace the Casimirs $\Pi^{(0)}, A_{+}^{(1)}$ by their chosen values and $A_{-}^{(1)}$ by its expression (3.6) through which $g$ is defined. This has no effect on the Poisson bracket of $\mathcal{L}(\lambda)$, which is therefore of the form (2.1) with $r_{\underline{12}}$ and $s_{\underline{12}}$ given explicitly later in (4.5). Thus, we have ${ }^{6}$

$$
\mathcal{L}(\lambda)=A^{(0)}+\frac{1}{2} \lambda^{-1} \mu_{-} g^{-1} T_{-} g-\frac{1}{2} \lambda \mu_{+} T_{+} .
$$

The expression for the temporal component $\mathcal{M}$ of the Lax pair similarly reads [25]

$$
\mathcal{M}(\lambda)=A^{(0)}+\ell-\frac{1}{2} \lambda^{-1} \mu_{-} g^{-1} T_{-} g-\frac{1}{2} \lambda_{\mu_{+}} T_{+} .
$$

The zero curvature equation $\left\{H^{\prime}, \mathcal{L}\right\}^{\prime}=\partial_{\sigma} \mathcal{M}+[\mathcal{M}, \mathcal{L}]$ is equivalent to the Hamiltonian equations of motion (3.28).

The upshot of this section is that the field theory obtained from the $F / G$ coset $\sigma$-model through the generalized Faddeev-Reshetikhin procedure is nothing but the one corresponding to the $G / H$ gauged WZW action with an integrable potential. This also means that we have automatically obtained the $r / s$-matrices associated with the Lax matrix of these latter models. In particular, their non-ultralocality is mild.

Another key point is that even though we have made a reduction, the Poisson brackets (3.11) on the reduced phase space is still perfectly local. The reason being that the reduction conditions of section 3.2 are Casimirs of the Poisson brackets (2.24). This is

\footnotetext{
${ }^{6}$ Here we restore the superscript notation corresponding to the grading $\mathfrak{f}=\mathfrak{f}^{(0)} \oplus \mathfrak{f}^{(1)}$.
} 
in sharp contrast with the result of applying a similar reduction to the canonical Poisson brackets (2.22) of the $\sigma$-model, where the corresponding Poisson brackets on the reduced phase space turn out non-local. For the $S^{2} \sigma$-model this has been worked out in [31] and references therein. In the case of string theory on $A d S_{5} \times S^{5}$, this was first studied in [32] and then in more detail in $[33,34]$.

\section{Towards a lattice discretization}

We have seen in the previous sections that a Faddeev-Reshetikhin type model could also be defined for coset $\sigma$-models. The important novelty in this case, however, is that the modified bracket (2.24) is also non-ultralocal. At first glance it might therefore seem that this new Poisson bracket is of no improvement compared to the original one. Indeed, the motivation for attempting to generalize the Faddeev-Reshetikhin approach to the case at hand was to try and do away with the problematic non-ultralocal terms occurring in the original brackets of the coset $\sigma$-model. Yet as we will show in the present section following [7], the non-ultralocality of the new bracket (2.24) is mild compared to that of the original Poisson bracket of the coset $\sigma$-model. In fact, quite remarkably, it turns out that with this milder form of non-ultralocality one is able to write down a corresponding well defined regularized lattice Poisson algebra which reduces to the non-ultralocal bracket of the Lax matrix in the continuum limit.

\subsection{Generalized Gauss decomposition}

As can be inferred from the form of the Poisson bracket (2.8) between functions of the Lax matrix, the non-ultralocality of our model stems from the fact that its $R$-matrix, defined as in (2.12), is not skew-symmetric with respect to the inner product at hand (2.23). Nevertheless, the crucial property which will ultimately enable us to discretize the Poisson bracket of Lax matrices corresponding to (2.24) is that the $R$-matrix only fails to be skew-symmetric on a finite-dimensional subalgebra of the full twisted loop algebra $\widehat{\mathfrak{f}}^{\sigma}[7]$. Specifically, the symmetric part of the $R$-matrix is a projection onto this subalgebra. In this case we say that the non-ultralocality of the resulting model is mild.

In order to describe the skew-symmetric and symmetric parts of $R$ with respect to (2.23), consider the following generalized Gauss decomposition

$$
\widehat{\mathfrak{f}}^{\sigma}=\widehat{\mathfrak{f}}_{<0}^{\sigma} \dot{+} \mathfrak{g} \dot{+} \widehat{\mathfrak{f}}_{>0}^{\sigma} \text {. }
$$

Recall the definitions of the projections $\pi_{<0}, \pi_{0}$ and $\pi_{>0}$ from section 2 . In terms of these, we may write the $R$-matrix (2.12) as $R=\pi_{>0}+\pi_{0}-\pi_{<0}$ while its skew-symmetric and symmetric parts respectively read

$$
r=\frac{1}{2}\left(R-R^{*}\right)=\pi_{>0}-\pi_{<0}, \quad s=\frac{1}{2}\left(R+R^{*}\right)=\pi_{0} .
$$

To see this, remember that the adjoint of the $R$-matrix with respect to the inner product $(2.23)$ is

$$
R^{*}=-\tilde{\lambda} \circ R \circ \tilde{\lambda}^{-1}
$$


Now the subspaces $\widehat{\mathfrak{f}}_{<0}^{\sigma}, \mathfrak{g}$ and $\widehat{\mathfrak{f}}_{>0}^{\sigma}$ respectively satisfy

$$
\lambda^{-1} \widehat{\mathfrak{f}}_{<0}^{\sigma} \subset \widehat{\mathfrak{f}}_{<0}, \quad \lambda^{-1} \mathfrak{g} \subset \widehat{\mathfrak{f}}_{<0}, \quad \lambda^{-1} \widehat{\mathfrak{f}}_{>0}^{\sigma} \subset \widehat{\mathfrak{f}}_{\geq 0} .
$$

Then decomposing any $X \in \widehat{\mathfrak{f}}^{\sigma}$ as $X=X_{<0}+X_{0}+X_{>0}$ according to (4.1) we have

$$
\begin{aligned}
R\left(\lambda^{-1} X_{>0}\right) & =\pi_{\geq 0}\left(\lambda^{-1} X_{>0}\right)=\lambda^{-1} X_{>0}=\lambda^{-1} \pi_{>0}\left(X_{>0}\right)=\lambda^{-1} R\left(X_{>0}\right), \\
R\left(\lambda^{-1} X_{0}\right) & =-\pi_{<0}\left(\lambda^{-1} X_{0}\right)=-\lambda^{-1} X_{0}=-\lambda^{-1} \pi_{0}\left(X_{0}\right)=-\lambda^{-1} R\left(X_{0}\right), \\
R\left(\lambda^{-1} X_{<0}\right) & =-\pi_{<0}\left(\lambda^{-1} X_{<0}\right)=-\lambda^{-1} X_{<0}=-\lambda^{-1} \pi_{<0}\left(X_{<0}\right)=\lambda^{-1} R\left(X_{<0}\right) .
\end{aligned}
$$

Now combining this result with (4.3) we see that the restriction of $R$ to the subspace $\widehat{\mathfrak{f}}_{<0}^{\sigma} \dot{+} \widehat{\mathfrak{f}}_{>0}^{\sigma}$ is skew-symmetric whereas its restriction to $\mathfrak{g}$ is symmetric, from which (4.2) follows.

Now let $\alpha \in$ End $\mathfrak{g}$ be any skew-symmetric solution of $\operatorname{mCYBE}(2.4)$ on $\mathfrak{g}$, with $\omega=1$. In particular $-\alpha$ is also a solution. It is straightforward to check by a direct calculation that the operators

$$
r \pm \alpha=\operatorname{diag}(-1, \pm \alpha, 1) \in \operatorname{End} \widehat{\mathfrak{f}}^{\sigma}
$$

where the diagonal decomposition is relative to (4.1), are both skew-symmetric solutions of $\mathrm{mCYBE}$ on $\widehat{\mathfrak{f}}^{\sigma}$. In other words we have

$$
[(r \pm \alpha) X,(r \pm \alpha) Y]-(r \pm \alpha)([(r \pm \alpha) X, Y]+[X,(r \pm \alpha) Y])+[X, Y]=0
$$

for any $X, Y \in \widehat{\mathfrak{f}}^{\sigma}$. It is important to stress that $r$ itself is not a solution of mCYBE. Furthermore one can also easily check that the matrices $s \pm \alpha$ satisfy the following relations with $r \pm \alpha$,

$$
[(s \pm \alpha) X,(s \pm \alpha) Y]=(s \pm \alpha)([(r \pm \alpha) X, Y]+[X,(r \pm \alpha) Y]) .
$$

Again we stress that if we set $\alpha=0$ these relations no longer hold.

One can write down explicit kernels for the operators $r \pm \alpha$ and $s \pm \alpha$ as follows. The kernels for the projection operators $\pi_{<0}, \pi_{0}$ and $\pi_{>0}$ respectively read

$$
\pi_{\underline{\mathbf{1 2}}}^{<0}(\lambda, \mu)=\sum_{m=1}^{\infty}\left(\frac{\mu}{\lambda}\right)^{m} C_{\underline{\mathbf{1 2}}}^{(m m)}, \quad \pi_{\underline{\mathbf{1 2}}}^{0}(\lambda, \mu)=C_{\underline{\mathbf{1 2}}}^{(00)}, \quad \pi_{\underline{\mathbf{1 2}}}^{>0}(\lambda, \mu)=\sum_{m=1}^{\infty}\left(\frac{\lambda}{\mu}\right)^{m} C_{\underline{\mathbf{1 2}}}^{(m m)} .
$$

It then follows that the kernels of the $r / s$ matices in (4.2) are [26]

$$
r_{\underline{\mathbf{1 2}}}(\lambda, \mu)=\frac{\mu^{2}+\lambda^{2}}{\mu^{2}-\lambda^{2}} C_{\underline{\mathbf{1 2}}}^{(00)}+\frac{2 \lambda \mu}{\mu^{2}-\lambda^{2}} C_{\underline{\mathbf{1 2}}}^{(1)}, \quad s_{\underline{\mathbf{1 2}}}(\lambda, \mu)=C_{\underline{\mathbf{1 2}}}^{(00)} .
$$

\subsection{Lattice algebra}

A standard way of constructing an integrable lattice discretization of a field theory on the circle is as follows. Recall that the zero curvature equation arises as the compatibility condition of the following auxiliary linear system

$$
\partial_{\sigma} \psi=\mathcal{L} \psi, \quad \partial_{\tau} \psi=\mathcal{M} \psi
$$


To discretize the spacial direction we replace the first equation by its discrete counterpart. This means breaking up the circle at a finite set of points $\sigma_{n} \in S^{1}, n=1, \ldots, N$ and considering the value of $\psi$ only at these points by defining $\psi_{n}=\psi\left(\sigma_{n}\right) \in \widehat{F}^{\sigma}$, where $\widehat{F}^{\sigma}$ is the loop group corresponding to $\widehat{\mathfrak{f}}^{\sigma}$. The lattice Lax matrix $\mathcal{L}^{n}$ is then defined to be the parallel transporter from the site $\sigma_{n}$ to the next site $\sigma_{n+1}$, namely

$$
\mathcal{L}^{n}=P \overleftarrow{\exp } \int_{\sigma_{n}}^{\sigma_{n+1}} \mathcal{L}(\sigma) d \sigma
$$

The spacial discretization of the auxiliary linear system (4.6) then takes the following form

$$
\psi_{n+1}=\mathcal{L}^{n} \psi_{n}, \quad \partial_{\tau} \psi_{n}=\mathcal{M}^{n} \psi_{n},
$$

where the second equation is obtained by evaluating the last equation of (4.6) at $\sigma_{n}$. We note here that $\mathcal{L}^{n}$ takes value in $\widehat{F}^{\sigma}$ whereas $\mathcal{M}^{n}$ still takes value in $\widehat{\mathfrak{f}}^{\sigma}$.

An important object in the continuum theory is the so called monodromy matrix $T$, defined as the parallel transporter around the full circle. It can be recovered on the lattice by multiplying all the lattice Lax matrices as

$$
T=\mathcal{L}^{N} \ldots \mathcal{L}^{1}
$$

The importance of this object stems from the fact that its spectral invariants $\operatorname{Tr}\left(T^{p}\right)$ generate integrals of motion of the continuum theory.

Having defined the lattice Lax matrices $\mathcal{L}^{n}$, the next step would be to determine their pairwise Poisson brackets. Unfortunately, recall from section 2.1.1 that when dealing with a non-ultralocal theory, the presence of $\delta_{\sigma \sigma^{\prime}}^{\prime}$ terms in the Poisson algebra of the continuum Lax matrix $\mathcal{L}(\sigma)$ prevents us from computing this directly. We therefore ask the reverse question, namely: does there exist a Poisson bracket $\left\{\mathcal{L}_{1}^{n}, \mathcal{L}_{2}^{m}\right\}^{\prime}$ satisfying all the necessary properties? First of all, this Poisson bracket should certainly be anti-symmetric and satisfy the Jacobi identity, i.e.

$$
\left\{\mathcal{L}_{\underline{\mathbf{1}}}^{n}, \mathcal{L}_{\underline{\mathbf{2}}}^{m}\right\}^{\prime}=-\left\{\mathcal{L}_{\underline{\mathbf{2}}}^{m}, \mathcal{L}_{\underline{\mathbf{1}}}^{n}\right\}^{\prime} \quad \text { and } \quad\left\{\mathcal{L}_{\underline{\mathbf{1}}}^{m},\left\{\mathcal{L}_{\underline{\mathbf{2}}}^{n}, \mathcal{L}_{\underline{\mathbf{3}}}^{p}\right\}^{\prime}\right\}^{\prime}+\text { cycl. }=0,
$$

for $m, n, p=1, \ldots, N$. Secondly, after requiring the Leibniz rule to hold, the resulting Poisson bracket of the monodromy matrix $T$ should be such that the integrals of motion are in involution. This means that

$$
\left\{\operatorname{Tr}\left(T^{p}\right), \operatorname{Tr}\left(T^{q}\right)\right\}^{\prime}=0,
$$

for any positive integers $p$ and $q$. The general quadratic Poisson algebra satisfying these requirements has been identified in $[36,37]$. However, the Poisson bracket between $\mathcal{L}^{n}$ and $\mathcal{L}^{m}$ should also reduce to the original Poisson algebra (2.1) in the continuum limit. Remarkably, it turns out that such a Poisson bracket does exist in the present case [7, 35]. It can be defined in terms of the matrices $r \pm \alpha$ and $s \pm \alpha$ as follows

$$
\begin{aligned}
\left\{\mathcal{L}_{\underline{\mathbf{1}}}^{n}, \mathcal{L}_{\underline{\mathbf{2}}}^{n}\right\}^{\prime} & =(r+\alpha)_{\underline{\mathbf{1 2}}} \mathcal{L}_{\underline{\mathbf{1}}}^{n} \mathcal{L}_{\underline{\mathbf{2}}}^{n}-\mathcal{L}_{\underline{\mathbf{1}}}^{n} \mathcal{L}_{\underline{\mathbf{2}}}^{n}(r-\alpha)_{\underline{\mathbf{1 2}}}, \\
\left\{\mathcal{L}_{\underline{\mathbf{1}}}^{n}, \mathcal{L}_{\underline{\mathbf{2}}}^{n+1}\right\}^{\prime} & =\mathcal{L}_{\underline{\mathbf{2}}}^{n+1}(s-\alpha)_{\underline{\mathbf{1 2}}} \mathcal{L}_{\underline{\mathbf{1}}}^{n}, \\
\left\{\mathcal{L}_{\underline{\mathbf{1}}}^{n}, \mathcal{L}_{\underline{\mathbf{2}}}^{m}\right\}^{\prime} & =0, \quad|n-m| \geq 2 .
\end{aligned}
$$


It has been shown in $[7,35]$ that $(4.10)$ is the unique algebra satisfying the above requirements. We refer the reader to $[7,35]$ for details and will content ourselves here with showing that the algebra (4.10) does indeed satisfy all the desired properties. First of all, we note that this is a well defined algebra [36, 37]. In particular, anti-symmetry follows using the (skew-)symmetry properties of $r, s$ and $\alpha$, and it satisfies the Jacobi identity by virtue of the relations (4.4) $[36,37]$. Next, using the Leibniz rule to compute the Poisson bracket of two monodromy matrices (4.8) we find

$$
\left\{T_{\underline{1}}, T_{\underline{2}}\right\}^{\prime}=(r+\alpha)_{\underline{12}} T_{\underline{1}} T_{\underline{2}}-T_{\underline{1}} T_{\underline{2}}(r-\alpha)_{\underline{12}}-T_{\underline{1}}(s+\alpha)_{\underline{12}} T_{\underline{2}}+T_{\underline{\mathbf{2}}}(s-\alpha)_{\underline{12}} T_{\underline{1}} .
$$

In deriving this relation, to cancel many terms we make essential use of the trivial but important fact that $(r+\alpha)_{\underline{\mathbf{1 2}}}+(s-\alpha)_{\underline{\mathbf{1 2}}}=(s+\alpha)_{\underline{\mathbf{1 2}}}+(r-\alpha)_{\underline{\mathbf{1 2}}}$. Recall also that we are assuming periodic boundary conditions. It is now easy to deduce from (4.11) that the involution property (4.9) holds.

Finally, we must show that (4.10) reduces to the correct continuum algebra of Lax matrices we started with when the lattice spacing goes to zero [7, 35]. For this we note that the lattice algebra (4.10) can equivalently be written as a single equation

$$
\begin{aligned}
\left\{\mathcal{L}_{\underline{\mathbf{1}}}^{n}, \mathcal{L}_{\underline{\mathbf{2}}}^{m}\right\}^{\prime}= & (r+\alpha)_{\underline{\mathbf{1 2}}} \mathcal{L}_{\underline{\mathbf{1}}}^{n} \mathcal{L}_{\underline{\mathbf{2}}}^{m} \delta_{m n}-\mathcal{L}_{\underline{\mathbf{1}}}^{n} \mathcal{L}_{\underline{\mathbf{2}}}^{m}(r-\alpha)_{\underline{\mathbf{1 2}}} \delta_{m n} \\
& -\mathcal{L}_{\underline{\mathbf{1}}}^{n}(s+\alpha)_{\underline{\mathbf{1 2}}} \mathcal{L}_{\underline{\mathbf{2}}}^{m} \delta_{m+1, n}+\mathcal{L}_{\underline{\mathbf{2}}}^{m}(s-\alpha)_{\underline{\mathbf{1 2}}} \mathcal{L}_{\underline{\mathbf{1}}}^{n} \delta_{m, n+1} .
\end{aligned}
$$

In order to take the continuum limit of this equation we write $\mathcal{L}^{n}=\mathbf{1}+\Delta \mathcal{L}\left(\sigma_{n}\right)+O\left(\Delta^{2}\right)$ where $\Delta=\sigma_{n+1}-\sigma_{n}$ is the lattice spacing. Substituting this into (4.12) and working to lowest order in $\Delta$ gives

$$
\begin{aligned}
\left\{\mathcal{L}_{\underline{\mathbf{1}}}\left(\sigma_{n}\right), \underline{\mathcal{L}_{\underline{\mathbf{2}}}}\left(\sigma_{m}\right)\right\}^{\prime}= & \Delta^{-1}\left[r_{\underline{\mathbf{1 2}}}, \mathcal{L}_{\underline{\mathbf{1}}}\left(\sigma_{n}\right)+\mathcal{L}_{\underline{\mathbf{2}}}\left(\sigma_{m}\right)\right] \delta_{m n} \\
& +\Delta^{-1} \mathcal{L}_{\underline{\mathbf{2}}}\left(\sigma_{m}\right) s_{\underline{\mathbf{1 2}}} \delta_{m, n+1}+\Delta^{-1} s_{\underline{\mathbf{1 2}}} \mathcal{L}_{\underline{\mathbf{1}}}\left(\sigma_{n}\right) \delta_{m, n+1} \\
& -\Delta^{-1} \underline{\mathcal{L}_{\underline{\mathbf{1}}}}\left(\sigma_{n}\right) s_{\underline{\mathbf{1 2}}} \delta_{m+1, n}-\Delta^{-1} s_{\underline{\mathbf{1 2}}} \mathcal{L}_{\underline{\mathbf{2}}}\left(\sigma_{m}\right) \delta_{m+1, n} \\
& -\Delta^{-1} \alpha_{\underline{\mathbf{1 2}}} \mathcal{L}_{\underline{\mathbf{2}}}\left(\sigma_{m}\right)\left(\delta_{m+1, n}-\delta_{m n}\right)+\Delta^{-1} \mathcal{L}_{\underline{\mathbf{2}}}\left(\sigma_{m}\right) \alpha_{\underline{\mathbf{1 2}}}\left(\delta_{m n}-\delta_{m, n+1}\right) \\
& +\Delta^{-1} \alpha_{\underline{\mathbf{1 2}}} \mathcal{L}_{\underline{\mathbf{1}}}\left(\sigma_{n}\right)\left(\delta_{m n}-\delta_{m, n+1}\right)-\Delta^{-1} \mathcal{L}_{\underline{\mathbf{1}}}\left(\sigma_{n}\right) \alpha_{\underline{\mathbf{1 2}}}\left(\delta_{m+1, n}-\delta_{m n}\right) \\
& -\Delta^{-2} \alpha_{\underline{\mathbf{1 2}}}\left(\delta_{m, n+1}-2 \delta_{m n}+\delta_{m+1, n}\right)-\Delta^{-2} s_{\underline{\mathbf{1 2}}}\left(\delta_{m+1, n}-\delta_{m, n+1}\right) .
\end{aligned}
$$

In the continuum limit $\Delta \rightarrow 0$ we let $\sigma_{n}=\sigma, \sigma_{m}=\sigma^{\prime}$ and make use of the following identities

$$
\begin{aligned}
\Delta^{-1} \delta_{m n} & \rightarrow \delta_{\sigma \sigma^{\prime}} \\
\Delta^{-1}\left(\delta_{m+1, n}-\delta_{m n}\right) & \sim \Delta \delta_{\sigma \sigma^{\prime}}^{\prime} \rightarrow 0, \\
\Delta^{-2}\left(\delta_{m+1, n}-2 \delta_{m n}+\delta_{m, n+1}\right) & \sim \Delta \delta_{\sigma \sigma^{\prime}}^{\prime \prime} \rightarrow 0, \\
\Delta^{-2}\left(\delta_{m+1, n}-\delta_{m, n+1}\right) & \rightarrow-2 \delta_{\sigma \sigma^{\prime}}^{\prime} .
\end{aligned}
$$

Note also that all the $O(1)$ terms in the above algebra vanish in this limit since they are multiplied by some $\delta_{m n}$ which effectively goes like $\Delta \delta_{\sigma \sigma^{\prime}}$. Taking $\Delta \rightarrow 0$ we therefore arrive at the following continuum algebra

$$
\left\{\mathcal{L}_{\underline{1}}(\sigma), \mathcal{L}_{\underline{\mathbf{2}}}\left(\sigma^{\prime}\right)\right\}^{\prime}=\left[r_{\underline{\mathbf{1 2}}}, \mathcal{L}_{\underline{1}}(\sigma)+\mathcal{L}_{\underline{\mathbf{2}}}(\sigma)\right] \delta_{\sigma \sigma^{\prime}}+\left[s_{\underline{12}}, \mathcal{L}_{\underline{\mathbf{1}}}(\sigma)-\mathcal{L}_{\underline{\mathbf{2}}}(\sigma)\right] \delta_{\sigma \sigma^{\prime}}+2 s_{\underline{\mathbf{1 2}}} \delta_{\sigma \sigma^{\prime}}^{\prime}
$$


which is precisely the Poisson algebra (2.1) of the Lax matrix $\mathcal{L}(\sigma)$. In particular, we notice that all dependence on the matrix $\alpha$ disappears in the continuum. Nevertheless, without $\alpha$ the lattice algebra we wrote down does not correspond to a well defined Poisson bracket of the $\mathcal{L}^{n}$ 's.

For consistency we should also check that the Poisson bracket of two parallel transporters with distinct end-points agrees with its direct computation since the latter is unambiguous [5]. Specifically, consider the product of lattice Lax matrices on successive sites

$$
T^{I, J}=\mathcal{L}^{I} \mathcal{L}^{I-1} \cdots \mathcal{L}^{J+1} \mathcal{L}^{J}
$$

for any $I>J$. This object is merely the parallel transporter from $\sigma_{J}$ to $\sigma_{I+1}$. The Poisson bracket

$$
\left\{T_{\underline{\mathbf{1}}}^{I, J}, T_{\underline{\mathbf{2}}}^{K, L}\right\}^{\prime}
$$

when all four $I, J, K, L$ are distinct may then be computed in two different ways: either using the lattice algebra (4.10) or by a direct computation. One may check that the result of the lattice calculation is independent of $\alpha$ and moreover it agrees with the result of the direct computation. This therefore shows that the matrix $\alpha$ only enters the Poisson brackets of parallel transporters which would otherwise be ill-defined.

The lattice algebra (4.10) is to be compared to its ultralocal counterpart (2.2). From the way the $s$-matrix appears in $(4.10 \mathrm{~b})$ it is clear that non-ultralocality manifests itself on the lattice by the fact that neighbouring lattice Lax matrices no longer Poisson commute. Note that the Poisson algebra (4.10) is precisely of the general quadratic $a b c d$-type discussed in $[36,37]$ if we let

$$
a_{\underline{12}}=(r+\alpha)_{\underline{12}}, \quad b_{\underline{12}}=(-s-\alpha)_{\underline{12}}, \quad c_{\underline{12}}=(-s+\alpha)_{\underline{12}}, \quad d_{\underline{12}}=(r-\alpha)_{\underline{12}} .
$$

\section{Comments and conclusion}

In this article we have generalized the first steps of the Faddeev-Reshetikhin procedure to symmetric space $\sigma$-models. Many comments come to mind.

To begin with let us go back to the case of the principal chiral model on a Lie group $G$. As we showed in section 2.2.2, for this model it is possible to completely rid the Poisson brackets of their non-ultralocality. The next step would then be to determine the new Hamiltonian. Following [6] this can be achieved by defining two functions $P_{S}$ and $P_{T}$ that act, with respect to the ultralocal Poisson brackets, as spatial derivative on $S=j_{0}+j_{1}$ and $T=j_{0}-j_{1}$ respectively. Although explicit expressions for $P_{S, T}$ can be obtained locally in terms of Darboux coordinates, these are less important at the quantum level where the operators $P_{S, T}$ are replaced by corresponding shift operators on the lattice. It might therefore be possible to also generalize the Faddeev-Reshetikhin procedure to a generic principal chiral model, even without having explicit classical expressions for the operators $P_{S, T}$.

It is however possible to proceed differently by treating the principal chiral model on $G$ as a symmetric space $\sigma$-model on $G \times G / G_{\text {diag }}$, where $G_{\text {diag }}$ denotes the diagonal subgroup. The work presented here may then be applied directly to this case, yielding the $G / \mathrm{U}(1)^{r}$ gauged WZW model with a potential where $r$ is the rank of $\mathfrak{g}$. These models are known 
as homogenous sine-Gordon models [38-42]. In particular, the case of the principal chiral model on $\mathrm{SU}(2)$ corresponds to the $\mathrm{SU}(2) / \mathrm{U}(1)$ gauged WZW model with a potential, which upon gauge fixing the $\mathrm{U}(1)$ invariance gives the complex sine-Gordon theory [8, 43-45]. An immediate drawback of this approach to treating the principal chiral model is that one departs from the analysis of [6] since the non-ultralocality is not completely removed. However, one advantage of proceeding in this way is that contrary to the case above, the action of these theories is explicitely Lorentz invariant. Furthermore, this puts the principal chiral model and the symmetric space $\sigma$-model on the same footing since the alleviation of non-ultralocality corresponds in both cases to a Pohlmeyer reduction.

Of course, the equivalence between the generalized Faddeev-Reshetikhin model defined in this article and the original symmetric space $\sigma$-model is restricted for the moment to the classical level. Any statement about the possible fate of this equivalence at the quantum level would be premature. In fact, this issue is already rather delicate for the lattice magnetic model defined in [6]. The quantization of this model by means of the Bethe ansatz describes excitations over the reference state, whereas the physical ground state is obtained by filling in the Dirac sea of Bethe roots. The claim made in [6] is that taking the classical limit around this physical ground state reproduces the non-ultralocal Poisson structure of the SU(2) principal chiral model. We refer the reader to the original article [6] as well as [46] and the more recent article [47] for tests of this claim. To proceed along the lines of [6] in the present case, the next challenge will be to explicitly construct a lattice model. The first step in this direction consists in writing the quantum lattice algebra corresponding to (4.11) which should be a quadratic algebra of the type discussed in [36, 37]. In this context, it would be desirable to investigate the connection with the so called lattice WZW model (see the set of lectures [48]) and Kac-Moody algebra introduced in [49, 50].

Independently of whether the program of generalizing the Faddeev-Reshetikhin procedure can be brought to its completion, an important byproduct of our work concerns the non-ultralocality of generalized sine-Gordon models. Although we have shown it in an indirect way, a prominent result of this article is that the non-ultralocality of such models, viewed as gauged WZW models with an integrable potential, is mild. To illustrate the significance of this result, let us focus on the complex sine-Gordon model as an example. When viewed as a gauged $\mathrm{SU}(2) / \mathrm{U}(1)$ WZW model plus a potential, the non-ultralocality of this model is mild. However, if we gauge fix the $\mathrm{U}(1)$ invariance then we obtain the complex sine-Gordon action

$$
\int d \sigma d \tau \frac{1}{2}\left(\frac{\left|\partial_{\mu} \psi\right|^{2}}{1-g^{2}|\psi|^{2}}-m^{2}|\psi|^{2}\right)
$$

For this action, the situation is completely different since the non-ultralocality of the corresponding Poisson structure is no longer mild and the associated $r$ and $s$ matrices are in fact dynamical [5]! This suggests that it may be preferable to try to discretize these theories at the level of the gauged WZW model with an integrable potential. This is reminiscent of the study in $[51,52]$ of the $S$-matrix of the complex sine-Gordon model, where the quantum counterterm added in [53-55] at one loop in order to maintain factorized scattering has a natural gauged WZW origin. 
One important motivation for the present work is of course related to the AdS/CFT correspondence [56-58] between superstring theory on $A d S_{5} \times S^{5}$ and $\mathcal{N}=4$ superconformal Yang-Mills theory (see [59] for a review). Since the non-ultralocality of the superstring on $A d S_{5} \times S^{5}$ is a major obstacle to quantizing this theory, it would certainly be very appealing if the Faddeev-Reshetikhin procedure could generalize to this context as well. In view of this one should start by extending the analysis presented here to semi-symmetric space $\sigma$ models. In fact, it has already been shown in [24] that the $r / s$ structure of the superstring on $A d S_{5} \times S^{5}$ uncovered in [60] has an algebraic origin which fits precisely into the $R$-matrix approach. This is exactly the right framework to proceed along the lines presented here. The analogue of the Poisson brackets (2.24) in this case is under investigation.

\section{Acknowledgments}

We would like to thank K. Gawedzki and J. M. Maillet for useful discussions. B.V. is extremely grateful to M. Semenov-Tian-Shansky for many valuable discussions. B.V. is supported by U.K. EPSRC grant EP/H000054/1.

\section{A Notations}

In this appendix we define some notations which are used throughout the text.

Given an operator $O$ acting on $\widehat{\mathfrak{f}}$, its kernel $O_{\underline{\mathbf{1 2}}}$ relative to the twisted inner product (2.6) is defined by

$$
(O X, Y)_{\varphi}=\left(O_{\underline{12}}, Y \otimes X\right)_{\varphi}, \quad \forall X, Y \in \widehat{\mathfrak{f}} .
$$

The kernel $O_{\underline{\mathbf{1 2}}}^{*}$ of the adjoint operator $O^{*}$ is then simply given by $O_{\underline{\mathbf{2 1}}}$.

When writing Poisson brackets in tensor notation we make use of the tensor Casimir $C_{\underline{\mathbf{1 2}}}$. It can be defined as the kernel of the identity operator id $\in$ End $\mathfrak{f}$ with respect to the inner product $\langle\cdot, \cdot\rangle$ on $\mathfrak{f}$. In other words it is defined by the following property

$$
\left\langle C_{\underline{12}}, x_{\underline{2}}\right\rangle_{2}=x_{\underline{1}},
$$

for any $x \in \mathfrak{f}$. It is easy to check that it satisfies the property $\left[C_{\underline{\mathbf{1 2}}}, x_{\underline{\mathbf{1}}}+x_{\mathbf{2}}\right]=0$. The corresponding property for any group element $g \in F$ reads

$$
g_{\underline{1}} g_{\underline{2}} C_{\underline{\mathbf{1 2}}}=C_{\underline{\mathbf{1 2}}} g_{\underline{1}} g_{\underline{2}} .
$$

When $\mathfrak{f}$ is equipped with an involution $\sigma: \mathfrak{f} \rightarrow \mathfrak{f}$ such that $\sigma^{2}=\mathrm{id}$, this induces a direct sum decomposition $\mathfrak{f}=\mathfrak{f}^{(0)} \oplus \mathfrak{f}^{(1)}$ into eigenspaces of $\sigma$. By the homomorphism property of $\sigma$, this $\mathbb{Z}_{2}$-grading has the property that

$$
\left[\mathfrak{f}^{(i)}, \mathfrak{f}^{(j)}\right] \subset \mathfrak{f}^{(i+j)}
$$

We shall always assume that the inner product $\langle\cdot, \cdot\rangle$ on $\mathfrak{f}$ respects the grading, in the sense that $\left\langle x^{(0)}, y^{(1)}\right\rangle=0$ for any $x^{(0)} \in \mathfrak{f}^{(0)}$ and $y^{(1)} \in \mathfrak{f}^{(1)}$. In this case the tensor Casimir can be decomposed as $C_{\underline{\mathbf{1 2}}}=C_{\underline{\mathbf{1 2}}}^{(00)}+C_{\underline{\mathbf{1 2}}}^{(1)}$ where $C_{\underline{\mathbf{1 2}}}^{(i i)} \in \mathfrak{f}^{(i)} \otimes \mathfrak{f}^{(i)}$. 
Open Access. This article is distributed under the terms of the Creative Commons Attribution License which permits any use, distribution and reproduction in any medium, provided the original author(s) and source are credited.

\section{References}

[1] L. Faddeev and L. Takhtajan, The quantum method of the inverse problem and the Heisenberg XYZ-model, Russ. Math. Surv. 34 (1979) 11.

[2] P. Kulish and E. Sklyanin, Quantum inverse scattering method and the Heisenberg ferromagnet, Phys. Lett. A 70 (1979) 461 [inSPIRE].

[3] L. Faddeev, E. Sklyanin, and L. Takhtajan, Quantum inverse problem method. I, Theor. Math. Phys. 57 (1980) 688.

[4] J.M. Maillet, Kac-Moody algebra and extended Yang-Baxter relations in the $O(N)$ nonlinear $\sigma$-model, Phys. Lett. B 162 (1985) 137 [INSPIRE].

[5] J.M. Maillet, New integrable canonical structures in two-dimensional models, Nucl. Phys. B 269 (1986) 54 [INSPIRE].

[6] L. Faddeev and N.Y. Reshetikhin, Integrability of the principal chiral field model in (1+1)-dimension, Annals Phys. 167 (1986) 227 [INSPIRE].

[7] M. Semenov-Tian-Shansky and A. Sevostyanov, Classical and quantum nonultralocal systems on the lattice, hep-th/9509029 [INSPIRE].

[8] K. Pohlmeyer, Integrable Hamiltonian systems and interactions through quadratic constraints, Commun. Math. Phys. 46 (1976) 207 [InSPIRE].

[9] K. Pohlmeyer and K.-H. Rehren, Reduction of the two-dimensional $O(n)$ nonlinear $\sigma$-model, J. Math. Phys. 20 (1979) 2628 [InSPIRE].

[10] H. Eichenherr and K. Pohlmeyer, Lax pairs for certain generalizations of the sine-Gordon equation, Phys. Lett. B 89 (1979) 76 [InSPIRE].

[11] H. Eichenherr, Infinitely many conserved local charges for the $C P^{N-1}$ models, Phys. Lett. B 90 (1980) 121 [INSPIRE].

[12] H. Eichenherr and J. Honerkamp, Reduction of the $C P^{n}$ nonlinear sigma model, J. Math. Phys. 22 (1981) 374 [INSPIRE].

[13] R. D'Auria, T. Regge and S. Sciuto, A general scheme for bidimensional models with associate linear set, Phys. Lett. B 89 (1980) 363 [INSPIRE].

[14] R. D'Auria, T. Regge and S. Sciuto, Group theoretical construction of two-dimensional models with infinite set of conservation laws, Nucl. Phys. B 171 (1980) 167 [INSPIRE].

[15] R. D'Auria and S. Sciuto, Group theoretical construction of two-dimensional supersymmetric models, Nucl. Phys. B 171 (1980) 189 [INSPIRE].

[16] V. Zakharov and A. Mikhailov, Relativistically invariant two-dimensional models in field theory integrable by the inverse problem technique (in Russian), Sov. Phys. JETP 47 (1978) 1017 [INSPIRE].

[17] I. Bakas, Q.-H. Park and H.-J. Shin, Lagrangian formulation of symmetric space sine-Gordon models, Phys. Lett. B 372 (1996) 45 [hep-th/9512030] [INSPIRE]. 
[18] M. Grigoriev and A.A. Tseytlin, Pohlmeyer reduction of $A d S_{5} \times S^{5}$ superstring $\sigma$-model, Nucl. Phys. B 800 (2008) 450 [arXiv:0711.0155] [InSPIRE].

[19] J.L. Miramontes, Pohlmeyer reduction revisited, JHEP 10 (2008) 087 [arXiv:0808.3365] [INSPIRE].

[20] P. Bowcock, Canonical quantization of the gauged Wess-Zumino model, Nucl. Phys. B 316 (1989) 80 [inSPIRE].

[21] M. Semenov-Tian-Shansky, What is a classical r-matrix?, Funct. Anal. Appl. 17 (1983) 259 [INSPIRE].

[22] M. Semenov-Tian-Shansky, Integrable systems and factorization problems, nlin/0209057.

[23] M. Semenov-Tian-Shansky, Integrable sytsems: the r-matrix approach, RIMS-1650, Research Institute for Mathematical Sciences, Kioto Japan (2008).

[24] B. Vicedo, The classical R-matrix of AdS/CFT and its Lie dialgebra structure, Lett. Math. Phys. 95 (2011) 249 [arXiv:1003.1192] [INSPIRE].

[25] B. Vicedo, Hamiltonian dynamics and the hidden symmetries of the $A d S_{5} \times S^{5}$ superstring, JHEP 01 (2010) 102 [arXiv:0910.0221] [INSPIRE].

[26] A. Sevostyanov, The classical $R$ matrix method for nonlinear $\sigma$-model, Int. J. Mod. Phys. A 11 (1996) 4241 [hep-th/9509030] [INSPIRE].

[27] A. Reyman and M. Semenov-Tian-Shansky, Compatible Poisson structures for Lax equations: an $R$ matrix approach, Phys. Lett. A 130 (1988) 456 [INSPIRE].

[28] J. Evans and A. Mountain, Commuting charges and symmetric spaces, Phys. Lett. B 483 (2000) 290 [hep-th/0003264] [INSPIRE].

[29] J.M. Evans, Integrable $\sigma$-models and Drinfeld-Sokolov hierarchies, Nucl. Phys. B 608 (2001) 591 [hep-th/0101231] [INSPIRE].

[30] E. Witten, On holomorphic factorization of WZW and coset models, Commun. Math. Phys. 144 (1992) 189 [inSPIRE].

[31] A. Mikhailov, A nonlocal Poisson bracket of the sine-Gordon model, J. Geom. Phys. 61 (2011) 85 [hep-th/0511069] [INSPIRE].

[32] A. Mikhailov, Bihamiltonian structure of the classical superstring in $A d S_{5} \times S^{5}$, Adv. Theor. Math. Phys. 14 (2010) 1585 [hep-th/0609108] [INSPIRE].

[33] D.M. Schmidtt, Supersymmetry flows, semi-symmetric space sine-Gordon models and the Pohlmeyer reduction, JHEP 03 (2011) 021 [arXiv: 1012.4713] [INSPIRE].

[34] D.M. Schmidtt, Integrability vs supersymmetry: Poisson structures of the Pohlmeyer reduction, JHEP 11 (2011) 067 [arXiv:1106.4796] [INSPIRE].

[35] M. Semenov-Tian-Shansky, Monodromy map and classical $R$ matrices, hep-th/9402054 [INSPIRE].

[36] L. Freidel and J. Maillet, Quadratic algebras and integrable systems, Phys. Lett. B 262 (1991) 278 [INSPIRE].

[37] L. Freidel and J. Maillet, On classical and quantum integrable field theories associated to Kac-Moody current algebras, Phys. Lett. B 263 (1991) 403 [INSPIRE]. 
[38] C.R. Fernandez-Pousa, M.V. Gallas, T.J. Hollowood and J.L. Miramontes, The symmetric space and homogeneous sine-Gordon theories, Nucl. Phys. B 484 (1997) 609 [hep-th/9606032] [INSPIRE].

[39] C.R. Fernandez-Pousa, M.V. Gallas, T.J. Hollowood and J.L. Miramontes, Solitonic integrable perturbations of parafermionic theories, Nucl. Phys. B 499 (1997) 673 [hep-th/9701109] [INSPIRE].

[40] J.L. Miramontes and C. Fernandez-Pousa, Integrable quantum field theories with unstable particles, Phys. Lett. B 472 (2000) 392 [hep-th/9910218] [INSPIRE].

[41] P. Dorey and J.L. Miramontes, Mass scales and crossover phenomena in the homogeneous sine-Gordon models, Nucl. Phys. B 697 (2004) 405 [hep-th/0405275] [INSPIRE].

[42] O.A. Castro-Alvaredo, Bootstrap methods in $1+1$ dimensional quantum field theories: The Homogeneous sine-Gordon models, hep-th/0109212 [INSPIRE].

[43] F. Lund and T. Regge, Unified approach to strings and vortices with soliton solutions, Phys. Rev. D 14 (1976) 1524 [INSPIRE].

[44] F. Lund, Example of a relativistic, completely integrable, hamiltonian system, Phys. Rev. Lett. 38 (1977) 1175 [INSPIRE].

[45] B. Getmanov, New Lorentz invariant systems with exact multi-soliton solutions, JETP Lett. 25 (1977) 119.

[46] C. Destri and H. de Vega, Light cone lattices and the exact solution of chiral fermion and $\sigma$-models, J. Phys. A 22 (1989) 1329 [InSPIRE].

[47] J. Caetano, Unified approach to the $\mathrm{SU}(2)$ principal chiral field model at finite volume, arXiv: 1012.2600 [INSPIRE].

[48] F. Falceto and K. Gawędzki, Lattice Wess-Zumino-Witten model and quantum groups, J. Geom. Phys. 11 (1993) 251 [hep-th/9209076] [INSPIRE].

[49] A. Alekseev, L. Faddeev, M. Semenov-Tian-Shansky and A. Volkov, The Unraveling of the quantum group structure in the WZNW theory, Preprint CERN-TH-5981/91 (1991).

[50] A. Alekseev, L. Faddeev and M. Semenov-Tian-Shansky, Hidden quantum groups inside Kac-Moody algebra, Commun. Math. Phys. 149 (1992) 335 [INSPIRE].

[51] N. Dorey and T.J. Hollowood, Quantum scattering of charged solitons in the complex sine-Gordon model, Nucl. Phys. B 440 (1995) 215 [hep-th/9410140] [INSPIRE].

[52] B. Hoare and A. Tseytlin, On the perturbative S-matrix of generalized sine-Gordon models, JHEP 11 (2010) 111 [arXiv: 1008.4914] [INSPIRE].

[53] H. de Vega and J. Maillet, Renormalization character and quantum S matrix for a classically integrable theory, Phys. Lett. B 101 (1981) 302 [InSPIRE].

[54] H. de Vega and J. Maillet, Semiclassical quantization of the complex sine-Gordon field theory, Phys. Rev. D 28 (1983) 1441 [INSPIRE].

[55] J. Maillet, Quantum U(1) invariant theory from integrable classical models, Phys. Rev. D 26 (1982) 2755 [INSPIRE].

[56] J.M. Maldacena, The large- $N$ limit of superconformal field theories and supergravity, Adv. Theor. Math. Phys. 2 (1998) 231 [Int. J. Theor. Phys. 38 (1999) 1113] [hep-th/9711200] [INSPIRE]. 
[57] S. Gubser, I.R. Klebanov and A.M. Polyakov, Gauge theory correlators from noncritical string theory, Phys. Lett. B 428 (1998) 105 [hep-th/9802109] [INSPIRE].

[58] E. Witten, Anti-de Sitter space and holography, Adv. Theor. Math. Phys. 2 (1998) 253 [hep-th/9802150] [INSPIRE].

[59] N. Beisert et al., Review of AdS/CFT integrability: an overview, Lett. Math. Phys. 99 (2012) 3 [arXiv: 1012.3982] [InSPIRE].

[60] M. Magro, The Classical Exchange Algebra of $A d S_{5} \times S^{5}$, JHEP 01 (2009) 021 [arXiv:0810.4136] [INSPIRE]. 